Published in final edited form as:

Nat Cell Biol. 2013 January ; 15(1): 50-60. doi:10.1038/ncb2652.

\title{
A Human Genome-wide Screen for Regulators of Clathrin-coated Vesicle Formation Reveals an Unexpected Role for the V-ATPase
}

\author{
Patrycja Kozik ${ }^{1}$, Nicola A. Hodson ${ }^{1}$, Daniela A. Sahlender ${ }^{1,2}$, Nikol Simecek ${ }^{1}$, Christina \\ Soromani $^{1,3}$, Jiahua $\mathbf{W u}^{1,4}$, Lucy M. Collinson ${ }^{5}$, and Margaret S. Robinson ${ }^{1}$ \\ ${ }^{1}$ Cambridge Institute for Medical Research, Wellcome Trust/MRC Building, University of \\ Cambridge, Hills Road, Cambridge CB2 OXY, UK \\ ${ }^{5}$ London Research Institute, Lincoln's Inn Fields Laboratories, 44 Lincoln's Inn Fields, London \\ WC2A 3LY, UK
}

\begin{abstract}
Clathrin-mediated endocytosis is essential for a wide range of cellular functions. We used a multistep siRNA-based screening strategy to identify regulators of the first step in clathrin-mediated endocytosis, formation of clathrin-coated vesicles (CCVs) at the plasma membrane. A primary genome-wide screen identified 334 hits that caused accumulation of CCV cargo on the cell surface. A secondary screen identified 92 hits that inhibited cargo uptake and/or altered the morphology of clathrin-coated structures. The hits include components of four functional complexes: coat proteins, V-ATPase subunits, spliceosome-associated proteins, and acetyltransferase subunits. Electron microscopy revealed that V-ATPase depletion caused the cell to form aberrant non-constricted clathrin-coated structures at the plasma membrane. The VATPase knockdown phenotype was rescued by addition of exogenous cholesterol, indicating that the knockdown blocks clathrin-mediated endocytosis by preventing cholesterol from recycling from endosomes back to the plasma membrane.
\end{abstract}

\begin{abstract}
Clathrin-mediated endocytosis (CME) is the pathway used by cells to internalize a variety of proteins and lipids. It is essential for processes as diverse as nutrient uptake, regulation of signalling by hormones and growth factors, and recycling of synaptic vesicle membranes. Although much of the machinery responsible for CME has been identified and characterised, the mechanisms that regulate CME are much less well understood. Several recent studies have emphasized the complexity of these regulatory mechanisms ${ }^{1-4}$. For instance, an siRNA library screen for genes involved in the endocytosis of transferrin and epidermal growth factor (EGF), both of which are taken up by CME, identified over 4,600 hits ${ }^{3}$. These genes most likely act at many different stages of the endocytic pathway, both upstream of clathrincoated vesicle (CCV) formation (e.g., transcription and translation of transferrin and EGF
\end{abstract}

\footnotetext{
Correspondence should be addressed to: P.K. (pk275@cam.ac.uk) or M.S.R. (msr12@mole.bio.cam.ac.uk) .

${ }^{2}$ Present address: Department for Fundamental Neurosciences, University of Lausanne, Rue du Bugnon 9, 1005 Lausanne, Switzerland

${ }^{3}$ Present address: Department of Clinical Biochemistry, University College London Hospitals, 60 Whitfield Street, London W1T 4EU, UK

${ }^{4}$ Present address: Medimmune, Granta Park, Cambridge, CB21 6GH, UK

Author contributions P.K. and M.S.R. designed the research; P.K. and C.S. carried out the genome-wide screen; P.K. and N.A.H. carried out the secondary screens; D.A.S. performed the thin sections analysis; D.A.S. and L.M.C. performed the "unroofing"; P.K. and N.A.H. performed the V-ATPase experiments; P.K., N.A.H., D.A.S., N.S., and J.W. analyzed data; P.K. and N.S. designed the webpage; P.K. and M.S.R. supervised the project; and P.K. and M.S.R. wrote the manuscript.

Competing Financial Interests The authors declare no competing financial interests.
} 
receptors) and downstream (e.g., trafficking between different types of endosomes and between endosomes and lysosomes).

Here, we set out to identify genes that specifically control the formation of CCVs at the plasma membrane (PM). We adopted a multi-step siRNA-based approach (Figure 1), involving plate reader-based assays to quantify the surface accumulation and internalization efficiency of model CCV cargo proteins, and a high-throughput microscopy-based assay to analyze the organization and morphology of clathrin-coated structures. Out of 92 top hits, we chose subunits of the V-ATPase for a more detailed analysis.

\section{RESULTS}

\section{Genome-wide screen: approach}

Inhibition of CME leads to the accumulation of clathrin-dependent cargo proteins on the cell surface. Hence, for the primary screen, we designed an assay to identify siRNAs from a human genome-wide library that cause an increase in the surface levels of two model CCV cargo proteins: CD8-YXXФ and CD8-FXNPXY. These two constructs contain the extracellular/lumenal and transmembrane domains of a $\mathrm{T}$ cell-specific protein, CD8, followed by very simple cytosolic tails containing the critical residues from either the YXXФ or the FXNPXY endocytic motif surrounded by alanines (Figure 1). We have previously shown that both of these constructs are efficiently endocytosed in a clathrindependent manner ${ }^{5}$, and for the present study, they were stably transfected into HeLa cells and expressed under the control of the human cytomegalovirus promoter. For the purpose of our screen, CD8-YXXФ and CD8-FXNPXY have two advantages over endogenous CCV cargo proteins: their expression is not affected by receptor-specific signalling pathways, and their trafficking relies on a single clathrin-dependent motif.

\section{Surface accumulation of CCV cargo}

The siRNA library used for the primary screen targets 20,052 genes with "SMARTpools" of four siRNAs, arrayed in 267 96-well plates. Every plate also contained both positive controls (CLTC (clathrin heavy chain) and AP2M1 (AP-2 $\mu$ subunit) siRNAs) and negative controls (PLK1 siRNA, no siRNA, and RISC-free siRNA) (Supplementary Figure S1). The siRNAs were reverse transfected into the CD8-YXXФ and CD8-FXNPXY cell lines in duplicate (i.e., 4 sets of plates in total). After $72 \mathrm{~h}$, the cells were fixed and immunostained for surface CD8 (Alexa ${ }^{488}$ ). To identify siRNAs causing non-clathrin-mediated changes in cell surface proteins ${ }^{6}$, the cells were also stained for the major histocompatibility complex (MHC) class I subunit, $\beta 2 \mathrm{~m}\left(\right.$ Alexa $\left.^{647}\right)$. Hoechst stain was used as an indicator of cell number (Figure 1a).

The fluorescence data for 52 spots across each well were collected in three channels using a plate reader (Figure 2a). Figure $2 \mathrm{~b}$ shows a plot of the Alexa ${ }^{488}$ and Hoechst readings from a sample plate on which there was a strong hit, DNM2 (dynamin 2). Plots of all the raw data can be found at http://www.bioinformatics.cimr.cam.ac.uk/siRNA/Robinson_Screen. After excluding any non-viable knockdowns from further analysis, the ratio of Alexa ${ }^{488}$ to Hoechst fluorescence was used as a measure of CD8 accumulation on the PM. To control for plate-to-plate variability in staining and knockdown efficiency, the Alexa ${ }^{488} / \mathrm{Hoechst}$ ratios from each plate were normalised to the positive (CTLC) and negative ("no knockdown") controls (Supplementary Figures S2-S3). There was a high correlation between the two technical repeats $\left(\mathrm{R}^{2}=0.94\right.$ for $\mathrm{YXX} \Phi, 0.84$ for FXNPXY) as well biological repeats $\left(\mathrm{R}^{2}=0.65\right.$ for YXX $\Phi, 0.89$ for FXNPXY, Supplementary Figure S4a,b).

We used strictly standardized mean difference (SSMD) ${ }^{7}$ to combine the duplicate measurements and rank SMARTpools according to both strength and reproducibility of 
phenotype (Figure 2c, Supplementary Figure S4c). An SSMD of greater than 3 was used as the threshold for hit selection. All of the hits for the CD8-YXXФ cells had at least 2.85 -fold more surface CD8 than the negative control, and all of the hits for the CD8-FXNPXY cells had at least 2.39-fold more surface CD8. For both cell lines, CLTC and DNM2 were among the top hits. AP2M1 was a strong hit for the CD8-YXXФ cells only. DAB2, one of two major FXNPXY adaptors, was a weak hit for the CD8-FXNPXY cells but below the selection threshold, consistent with reports that both DAB2 and ARH have to be knocked down simultaneously to prevent internalization of FXNPXY-containing proteins ${ }^{8}$. As expected, several SMARTpools caused an increase of both CD8-YXXФ and CD8-FXNPXY $\left(\mathrm{R}^{2}=0.47\right)$. However, there was no correlation between the CD8 constructs and MHC-I $\left(\mathrm{R}^{2}\right.$ $=0.01$ ) (Figure 2d). Table S1 lists the 719 SMARTpools with SSMDs $>3$ for either or both of the motifs.

\section{Selection of hits for secondary assays}

In order to eliminate some of the potential false positives, we applied three exclusion criteria to the primary hit list (Figure 2e). First, the siRNA targets were re-annotated according to version 25 of the RefSeq database, and where multiple or no target mRNAs were identified, a SMARTpool was excluded from the hit list. Second, we performed microarray analyses and excluded hits for which the target mRNA was represented on the microarray but not detected. Finally, we excluded hits where the increase of CD8 on the cell surface was accompanied by an increase in MHC-I, as these genes are unlikely to control CME. Out of the 334 hits remaining, the 241 top hits (Table S1a) were selected for secondary screens, in which we used a set of different siRNA pools, chemically modified to reduce the off-target activity and designed with improved sequence selection algorithms (ONTARGETplus, OTP $)^{9,10}$.

\section{Efficiency of CME}

To confirm that knockdowns causing an increase in surface levels of CD8 specifically affect endocytosis, we determined the internalization efficiency of the CD8 constructs using a previously described antibody uptake assay ${ }^{5}$, adapted here for high-throughput analysis with a plate reader and shown diagrammatically in Figure 1b. Briefly, the siRNA-transfected CD8-YXXФ and CD8-FXNPXY cells were first allowed to internalize an anti-CD8Alexa ${ }^{488}$ antibody for $40 \mathrm{~min}$ at $37^{\circ} \mathrm{C}$. The cells were then fixed, surface Alexa ${ }^{488}$ fluorescence was quenched with an anti-Alexa ${ }^{488}$ antibody, and surface CD8 was relabelled with an Alexa ${ }^{647}$ secondary antibody (Figure 3a). The ratio of green to red fluorescence normalised to positive and negative controls in each plate was then used as a measure of internalization efficiency ( 0 corresponds to inhibition in CLTC knockdown cells, and 1 to uptake efficiency in "no knockdown" controls) (Figure 3b). 28 SMARTpools inhibited internalization of YХХФ, 38 of FXNPXY, and 20 of these were shared between the two cargo proteins (Figure $3 b$ ).

\section{Morphology of CCSs at the PM}

To identify hits that affect the formation of endocytic CCVs, we designed an automated microscopy-based assay to characterize clathrin-coated structures (CCSs) at the PM. We labelled the CCSs with an antibody against CALM, an accessory protein that colocalizes with clathrin at the PM, but not on intracellular membranes ${ }^{11,12}$ (see Figure 1c). The prerequisite for quantitative high throughput microscopy is obtaining good quality images, and in the case of PM CCSs, the optimal focal plane is where cells adhere to the growth surface. Thus, as a reference, we used hydrophilic Cy5-labeled flow cytometry beads, which settle between the cells at the bottom of the dish. Whole cell stain (WCS) was used to demark cell boundaries, and a SpotDetector BioApplication was used to identify CCSs in each cell (Figure 4a). 
We screened the 241 selected siRNA pools by reverse transfecting the cells in duplicate as before, then splitting the plates into four different densities after $48 \mathrm{~h}$, and fixing and staining the cells after $72 \mathrm{~h}$. The analysis was performed in two independent experiments with 300-2000 valid cells analyzed for each knockdown (Table S2). Mitotic cells and cells from dense fields were excluded from the analysis (Figure $4 b, c)$. We focused on changes in total fluorescence intensity per spot and spot count per area (Figure 4d,e,f) and selected 67 hits falling into three categories: increase in spot brightness (red), decrease in spot brightness (blue), and decrease in spot count without a change in brightness (yellow).

\section{Functional networks within genes that affect CCS morphology and CME efficiency}

The 92 hits combined from the two secondary assays (22 of which were shared hits) were analyzed using the STRING database ${ }^{13}$. Four groups of functionally or genetically linked genes were identified (Figure 5). CLTC, DNM2, and AP2M1 were correctly assigned to one network. The remaining three networks comprise: 1) subunits of the vacuolar ATPase (VATPase) (ATP6V0C, ATP6V0E, ATP6V1B2, ATP6V1D, ATP6V1F), 2) genes involved in mRNA splicing (CDC5L, CDC40, SFRS3, SF3B3, SF3B4, SFPQ, SFRS3), and 3) the histone acetyltransferase complex (RUVBL2, EP400, HTATIP (KAT5)). We decided to focus on V-ATPase for the present study.

\section{Ultrastructure of CCSs following depletion or inhibition of V-ATPase}

Although V-ATPase plays an important role in endocytic trafficking by controlling the intralumenal $\mathrm{pH}$ of endosomes and lysosomes, a role in the formation of CCVs at the PM was unexpected. siRNAs against eight components of V-ATPase were present among the primary hits, and using the OTP pools, we verified that the knockdowns also inhibit internalization of an endogenous ligand, transferrin (Figure 6a). To investigate the ultrastructure of the "brighter" CCSs observed in V-ATPase-depleted cells (Figure 6b), we turned to electron microscopy. Strikingly, knocking down V-ATPase caused the cells to accumulate large and irregular CCPs with average neck diameters of $220 \mathrm{~nm}$ (compared to $97 \mathrm{~nm}$ in control cells), while constricted pits were notably absent (Figure 6c,d,e).

The inability of pits to constrict and the block in CME upon V-ATPase depletion were surprising, as acute inhibition of the V-ATPase with Bafilomycin A1 (BafA1) does not block transferrin internalization, but instead inhibits its recycling ${ }^{14}$ (Figure 6f). However, when we incubated the cells with BafA1 for $24 \mathrm{~h}$, transferrin internalization was completely abolished (Figure 6f). This decrease in the efficiency of internalization occurred progressively over the $24 \mathrm{~h}$ period, and resulted in a gradual accumulation of the transferrin receptor at the cell surface (Figure 6g). In a similar time-course experiment, we observed a corresponding increase in the brightness of CALM-labelled CCSs (Figure 6h). EM analysis revealed that at $17 \mathrm{~h}$, BafA1-treated cells also accumulate wide, non-constricted CCSs (Figure $6 \mathrm{i}, \mathrm{j}, \mathrm{k}$ ). Together, these data suggest that siRNA-mediated depletion of different $\mathrm{V}$ ATPase subunits, or inhibition of V-ATPase activity with BafA1, block CME by preventing CCPs from forming CCVs.

\section{V-ATPase inhibition and cytosolic acidification}

What is the role of V-ATPase in CCP constriction and vesicle budding? Because V-ATPase is a proton pump ${ }^{15}$ and clathrin polymerization has been shown to be sensitive to $\mathrm{pH}^{16}$, we suspected initially that BafA1 treatment might prevent CCV formation by decreasing the $\mathrm{pH}$ of the cytosol. Studies by Heuser on "unroofed" cells have shown that cytosol acidification leads to an accumulation of "arrested" CCSs surrounded by membraneless microcages ${ }^{16}$. Using a similar technique, we generated PM replicas from control and BafA1-treated cells to examine their three-dimensional architecture. The micrographs confirmed the abundance of 
enlarged CCSs and revealed an astounding variety in their morphologies (Figure 7a,b). However, we did not see any evidence of microcage formation.

Two other treatments that lead to a block in endocytosis coupled with microcage formation, hypertonic sucrose and potassium depletion ${ }^{17}$, simultaneously block clathrin exchange ${ }^{18}$. Therefore, we performed FRAP experiments on cells transfected with GFP-tagged clathrin light chain, cultured in the presence or absence of BafA1 (Figure 7c,d). BafA1 treatment did not significantly affect the recovery kinetics, again arguing that clathrin is unlikely to be "locked" in microcages.

Finally, we measured the cytosolic and submembranous $\mathrm{pH}$ using soluble and SuperEcliptic (SE) pHluorin-mCherry proteins ${ }^{19}$ (Figure 7e). Since SEpHluorin fluorescence decreases upon protonation, the ratio of green to red signal can be used to estimate $\mathrm{pH}$, as demonstrated by incubating the cells in buffers of different $\mathrm{pH}$ in the presence of an ionophore, nigericin (Figure $7 \mathrm{f}, \mathrm{g}$ ). Treatment with BafA1 for $24 \mathrm{~h}$ resulted in only a modest $\mathrm{pH}$ decrease. Because $\mathrm{pH}$ 6.5-6.8 has been consistently reported as the threshold at which CME is inhibited ${ }^{19,20}$, we concluded that changes to cytosolic $\mathrm{pH}$ and microcage formation are unlikely to explain CME inhibition in the V-ATPase-depleted cells.

\section{CCV formation in cells lacking functional V-ATPase can be rescued with soluble cholesterol}

If the inhibition of CCV formation upon V-ATPase inhibition cannot be attributed to a decrease in cytosolic $\mathrm{pH}$, could it be related to the alkalization of endosomes? It has been known for over 30 years that if endosomes cannot acidify, trafficking out of them is inhibited $^{21}$, and this is thought to be due to a general block in the formation of carriers ${ }^{22}$. If an essential CCV membrane component were unable to recycle back to the plasma membrane, this would help to explain the BafA1 kinetics, because it would take time for this component to be depleted. A candidate for the "missing component" emerged from the observation that the morphology of the CCSs in V-ATPase-depleted cells is strikingly similar to the enlarged, shallow clathrin-coated invaginations that form upon extraction of cholesterol from the PM with methyl- $\beta$-cyclodextrin ${ }^{23,24}$. Like the transferrin receptor, cholesterol recycles from endosomes to the $\mathrm{PM}^{25}$, and consequently, in the presence of BafA1 it accumulates in endosomes and is depleted from the cell surface 26,27 . We found that if we either knocked down V-ATPase or treated cells with BafA1 for $24 \mathrm{~h}$, cholesterol (labelled with filipin) was lost from the PM and accumulated in an intracellular compartment, and we were able to quantify this change in three different cell lines using an automated microscopy-based assay (Fig. 8a,b). Double labelling indicates that the filipinpositive compartment also contains trapped CCV cargo proteins (Supplementary Figure S6). To test whether the loss of PM cholesterol could explain the block in endocytosis, we added exogenous cholesterol to the cells together with BafA1 or siRNAs targeting V-ATPase (Supplementary Figure S7). We found that transferrin internalization was partially rescued (Figure 8c,d), and there was also a rescue of CCS ultrastructure in PM replicas (Figure 8e,f) and a corresponding decrease in CCS brightness (Figure 8g).

\section{DISCUSSION}

To identify regulators of CCV formation, we carried out a multi-step siRNA-based screen. In the primary screen, hits were defined as siRNA pools that caused CCV cargo proteins with YXXФ or FXNPXY motifs to accumulate on the PM. Using further selection criteria based on gene expression and siRNA pool specificity, we narrowed down the list to 334 hits. Overall, the hit selection criteria applied are highly stringent and therefore may produce false negatives. However, this strategy allowed us to identify a small number of high confidence hits, which we characterized in more detail. 
To identify genes that control endocytosis at the step of CCV formation, we performed two secondary screens. First, we carried out an uptake assay, which allowed us to distinguish knockdowns that decrease the efficiency of CME from those than increase surface levels of CD8 for other reasons (e.g., inefficient lysosomal degradation). Second, we carried out a microscopy-based assay, which allowed us to identify knockdowns that alter the morphology of CCSs at the PM. Because the intensity of CALM fluorescence peaks during vesicle scission ${ }^{28}$, an increase in spot brightness could correspond to an accumulation of deeply invaginated pits ${ }^{29}$ (e.g., in dynamin-depleted cells ${ }^{30}$ ), or to an accumulation of unusually large or clustered pits. A decrease in spot count or brightness suggests inefficient coat nucleation or recruitment ${ }^{31}$.

From the final set of 92 hits, only CLTC, AP2M1, and DNM2 have been detected in CCVs by proteomics ${ }^{32}$. This is, however, not surprising considering that individual knockdowns of accessory $\mathrm{CCV}$ components (e.g. $\mathrm{CALM}^{33}$ or epsins ${ }^{34}$ ) rarely lead to endocytic arrest. Thus, the siRNA-based approach appears to be biased towards proteins not physically incorporated into CCVs. Other proteins implicated in CCV formation, such as FCHo1/2 and Rab5A/B/C, were also not picked up as strong hits, possibly because they are encoded by functionally redundant genes (this functional redundancy also helps to explain why only one of the four AP-2 subunits was present in the list).

Although the purpose of this screen was to identify regulators of clathrin-mediated trafficking, the data we have generated can be mined to look for genes involved in other pathways as well. For instance, knockdowns that decrease surface expression of a particular protein may affect its secretion and/or recycling. In addition, for each knockdown, the MHC-I results are also available online and will be an interesting dataset to explore, especially because post-Golgi trafficking pathways of MHC-I are poorly characterized ${ }^{6}$ and $30 \%$ of MHC-I hits correspond to unknown proteins. All of our data can be accessed at http://www.bioinformatics.cimr.cam.ac.uk/siRNA/Robinson_Screen.

Out of our 92 hits, several are uncharacterised proteins, such as HLXB9 and LOC124220, which we have now validated with two or more distinct siRNAs, and which we intend to characterise more fully. Other hits have been at least partially characterised, and this enabled us to group them into three functional networks (plus the network containing coat components). One network consists of several components of the mammalian spliceosome. Interestingly, a number of $\mathrm{CCV}$ proteins exist in multiple splice variants, including the a and $\beta$ subunits of $\mathrm{AP}-2^{35}$, clathrin light chains ${ }^{36}$, and dynamin $2^{37,38}$. The spliceosome is also essential for cell cycle progression ${ }^{39}$, and since endocytosis is arrested in mitotic cells ${ }^{40}$, further experiments will be required to resolve whether the endocytic block in spliceosome-depleted cells is due to incorrect splicing of coat proteins, or is a consequence of the mitotic arrest.

The second unexpected functional cluster contains subunits of the histone acetyltransferase complex. Histone acetylation plays an important role in the regulation of gene expression; however, three lines of evidence suggest that the block in CME may be due to changes in the acetylation of cytoplasmic proteins rather than histones. First, acetylation sites have been identified in three core components of CCVs: clathrin, AP-2, and dynamin $2^{41}$. Second, both RUVBL2 and KAT5 can translocate to the cytoplasm under certain conditions ${ }^{42-44}$. Third, in a hepatic cell line, WIF-B, a 30 min treatment with trichostatin A, a deacetylase inhibitor, was found to arrest deeply invaginated pits prior to fission ${ }^{45}$, and the short time scale argues against this effect being due to transcriptional regulation. Finding the key target(s) of the acetyltransferase complex will be critical for deciphering the role of acetylation in CME. 
The third unexpected network, which we analysed in more detail, contains several VATPase subunits. We discovered that the block in CCV formation in V-ATPase-depleted cells can be explained by the retention of cholesterol in non-acidified endosomes, and its concomitant loss from the PM. At first glance, this phenotype is reminiscent of NiemannPick type $\mathrm{C}$ disease, where mutations in $\mathrm{pH}$-sensitive NPC1 or NPC2 lead to accumulation of cholesterol in endosomes and lysosomes ${ }^{46,47}$. However, in NPC cells, cholesterol is still exchanged with the $\mathrm{PM}^{48,49}$ and endocytosis still occurs ${ }^{50}$. In contrast, when endosomes are unable to acidify, trafficking out of them becomes inhibited. Although this phenomenon was first observed in the $1980 \mathrm{~s}^{21}$ it still awaits a molecular explanation, but endosome acidification is clearly important for the recycling of lipids as well as proteins.

Why is cholesterol needed for CCV formation? Since transbilayer diffusion of cholesterol is fast $(<1 \mathrm{~s})^{51,52}$ relative to the half-time of CCV formation $(\sim 100 \mathrm{~s})^{28}$, it is possible that the spontaneous flip-flop of cholesterol could simply lower the membrane-bending energy. Thus, although the coat is still able to curve the membrane into a CCP in the absence of cholesterol, the energy required for the neck to constrict may be too great ${ }^{52}$ (Figure $8 \mathrm{~h}$ ). In yeast, budding of CCVs from intracellular membranes requires a lipid flippase, Drs2p, consistent with the notion that the distribution of lipids between the two leaflets of the bilayer is important for membrane deformation ${ }^{53}$.

The role of V-ATPase in CME is particularly relevant in light of recent therapeutic strategies against cancer that target V-ATPase activity ${ }^{54}$. Interestingly, one such study demonstrated that BafA1-induced inhibition of cell growth can be rescued with cholesterol or iron ${ }^{55}$. The authors assumed that receptor-mediated endocytosis was still occurring normally in the BafA1-treated cells, but that iron and cholesterol were unable to be released in endosomes and lysosomes. However, the present study demonstrates that long-term BafA1 treatment inhibits CME, and this might account, at least in part, for the cytostatic effects of the V-ATPase inhibitors. V-ATPase activity has also been implicated in signalling by both Notch ${ }^{56,57}$ and $\mathrm{Wnt}^{58,59}$. Although again it was assumed that the molecules were still getting internalised, and that the phenotype was caused by $\mathrm{pH}$-dependent events in the endosome, it is important to note that the Notch and Wnt pathways both require clathrin and dynamin ${ }^{60-63}$. Thus, the connection we have uncovered between endosome acidification, cholesterol trafficking, and CCV formation may help to explain a number of observations on the role of V-ATPase in growth control and development.

\section{MATERIALS AND METHODS}

\section{Antibodies, plasmids and other reagents}

The following antibodies were used in the primary screen: mouse anti-CD8 (1:500, ATCC number: CRL-8014, LGC Promochem), rabbit anti- $\beta 2 \mathrm{~m}$ (A0072, Dako Cytomation, 1:500), Alexa $^{488}$-labelled donkey anti-mouse IgG (A21202, 1:500) and Alexa ${ }^{647}$-labelled goat antirabbit IgG (A21244, 1:500) (Invitrogen); in the CCS assay: goat anti-CALM C-18 (sc-6433, Santa Cruz Biotechnology, 1:200), Alexa ${ }^{488}$-labelled donkey-anti-goat IgG (A11055, Invitrogen, 1:400); in the CD8 uptake assays: Alexa ${ }^{488}$-labeled anti-CD8 (MCA1226A488, Serotec, 1:50), anti-Alexa ${ }^{488}$ (A11094, Invitrogen, 1:67), anti-mouse IgG Alexa ${ }^{647}$ (A31571, Invitrogen, 1:400); in the transferrin uptake assays: anti-transferrin receptor (555534, BD Pharminogen 1:200).

${ }^{125}$ I-labeled transferrin was purchased from Perkin Elmer-Cetus. Alexa ${ }^{488}$-labelled transferrin (Invitrogen) was made up according to manufacturer's instructions and used at 20 $\mu \mathrm{g} / \mathrm{ml}$. Hoechst 33342 (Invitrogen) was used at $10 \mu \mathrm{g} / \mathrm{ml}$. Cellomics ${ }^{\circledR}$ Whole Cell Stain (WCS) Blue (CCSs assay) and Green (filipin assay) (Thermo Scientific) were prepared according to the manufacturer's instructions and used at 1:250 dilution. LysoTracker® Red 
DND-99 (Invitrogen) was added to the cells at a final concentration of $50 \mathrm{nM}$ for $5 \mathrm{~min}$ prior to live-cell imaging.

Bafilomycin A1 (Sigma-Aldrich) was dissolved in DMSO and used at a final concentration of $100 \mathrm{nM}$; corresponding volume of DMSO (1:1000, Sigma) was added as control. Watersoluble cholesterol (40 mg/g material, balanced with M $\beta C D$, Sigma-Aldrich) was dissolved in $\mathrm{H}_{2} \mathrm{O}$ and added to the cells at concentrations indicated in the text.

Filipin complex (F9765, Sigma) was dissolved in DMSO on the day, and used at a concentration of $25 \mu \mathrm{g} / \mathrm{ml}(1: 1000)$.

The pHluorin-mCherry plasmids (cytosolic and PM-targeted) were a kind gift from Sergio Grinstein ${ }^{19}$. The CD63-pEGFP C2 plasmid was a kind gift from Paul Luzio. The GFPclathrin light chain A plasmid was a kind gift from Steve Royle (University of Liverpool). The pHluorin-transferrin receptor plasmid was a kind gift from Christien Merrifield (LEBS, Gif-sur-Yvette, France).

\section{SiRNAs}

The human genome-wide siRNA library and other siRNAs were purchased from Dharmacon (Thermo-Fisher Scientific). The library contains 21,121 siGENOME SMARTpools (four siRNAs per gene) arrayed in 96-well plates; 20,052 SMARTpools were unique, 65 were duplicated within the library, and two were present in three different positions (these SMARTpools were used to estimate biological reproducibility). 241 ONTARGETplus SMARTpools were used for secondary screens (Supplementary Table S2). siGENOME SMARTpools against CLTC (M-004001-00), AP2M1 (M-008170-00), and PLK1 (M-003290-01), chemically modified siRNA, impaired in uptake and processing by RISC (RISC-free, D-001220-01), and a non-targeting pool (NTP, D-001810-10) were used as controls.

\section{Cell culture and DNA transfections}

The HeLa-M cells ${ }^{64}$ used throughout the study were grown in Dulbecco's Modified Eagle's medium (D6546, DMEM) supplemented with 10\% fetal bovine serum (FBS), 2 mM Lglutamine, $100 \mathrm{U} / \mathrm{ml}$ penicillin and $100 \mu \mathrm{g} / \mathrm{ml}$ streptomycin (Sigma-Aldrich). The Hela-M cells expressing CD8-YXXФ or CD8-FXNPXY constructs were previously described ${ }^{5}$. Retinal pigmented epithelium (RPE) cells were grown in Nutrient Mixture F-12 Ham mixed 1:1 with DMEM containing $2 \mathrm{mM}$ L-glutamine, and supplemented with $10 \% \mathrm{FBS}, 100 \mathrm{U} / \mathrm{ml}$ penicillin and $100 \mu \mathrm{g} / \mathrm{ml}$ streptomycin (Sigma-Aldrich). The A431 cells were grown in DMEM GlutaMAX-I (Gibco) with 10\% FBS, $100 \mathrm{U} / \mathrm{ml}$ penicillin and $100 \mu \mathrm{g} / \mathrm{ml}$ streptomycin (Sigma-Aldrich). All cells were grown at $37^{\circ} \mathrm{C}$ with $5 \% \mathrm{CO}_{2}$.

For DNA transfections, cells were grown to 50\% confluency and transfected for $24 \mathrm{~h}$ using a TransIT-HeLaMONSTER Transfection Kit (Mirus) with $2 \mu \mathrm{g}$ plasmid DNA per 6-well.

\section{mRNA analysis}

RNA was isolated from $10^{6} \mathrm{CD} 8-\mathrm{YXX} \Phi$ or CD8-FXNPXY cells (harvested by trypsinization) using the Purescript ${ }^{\circledR}$ RNA Purification Kit (Life Sciences Germany). Samples were processed and analyzed at the Centre for Microarray Resources (Department of Pathology, University of Cambridge). The analysis was performed using Illumina HumanRef-8.v2 BeadChip array scanned on Illumina BeadStation 500 platform. The list of expressed genes was generated using low stringency criteria to minimize the number of false negatives. Variance Stabilizing Transform and Robust Spline Normalization algorithms ${ }^{65}$ from the lumi R package ${ }^{66}$ were used to process the signal intensity data. 12,269 probes 
with signal intensity above the background in at least one of the technical replicates of either of the cell line were selected using a p-value threshold of $<0.01$ (listed in Supplementary Table S3). MIAME-compatible data was submitted to GEO (accession: GSE39954).

\section{SiRNA reverse transfection protocol}

A 96-well plate with $10 \mu \mathrm{l}$ of $1 \mu \mathrm{M}$ pre-aliquoted siRNAs was used to generate 4 assay plates (Viewplates, Cat. No. 6005182, PerkinElmer) (see Supplementary Figure S1). First, $72 \mu 1$ of Optimem I (Invitrogen) was added into each well of the transfection plate. Oligofectamine (Invitrogen) was pre-incubated with Optimem in a 1:8 ratio for $8 \mathrm{~min}$, and $18 \mu \mathrm{l}$ of the mix was added to each well. The transfection mix was incubated for $20 \mathrm{~min}$ at $\mathrm{RT}$, and aliquoted into assay plates ( $20 \mu \mathrm{l}$ per well). Next, 4500 cells in $100 \mu 1$ of DMEM (with $\mathrm{P} / \mathrm{S}$ and L-glutamine) were added to each well, and the plates were gently vortexed, then incubated at $37^{\circ} \mathrm{C}$ in $5 \% \mathrm{CO}_{2}$ for $72 \mathrm{~h}$. For knockdowns in 6-well plates, the volumes per well were scaled up 20x.

\section{Genome-wide and secondary screens}

Detailed protocols, methods for data analysis, and hit selection strategy are provided in the Supplementary Note.

\section{Cholesterol redistribution assay}

The cells were seeded in the 96-well plates $24 \mathrm{~h}$ before the analysis and fixed in 3.3\% formaldehyde in PBS for $30 \mathrm{~min}$ at room temperature. The cells were then washed in PBS and stained with filipin (1:1000 in PBS) and WCS Green for $2 \mathrm{~h}$. Cy5 beads (see CCS assay) in PBS were added before imaging. The images were acquired on the Arrayscan platform using beads as the focusing reference. Filipin was imaged at two focal plates: offset: $-4 \mu \mathrm{m}$ and $-1 \mu \mathrm{m}$, and the WCS was imaged without an offset. The images were then analyzed using a SpotDetector Bioapplication. PM cholesterol was quantified using the image with -4 $\mu \mathrm{m}$ offset where fluorescence was quantified within the "ring" region, and intracellular cholesterol was quantified using the image with $-1 \mu \mathrm{m}$ offset where fluorescence was quantified within the perinuclear "circular" region. Total fluorescence of the "spots" per object within the "ring" region was used to estimate cholesterol loss from the PM. On the scatter plot in Figure 8b, the amount of the intracellular cholesterol was estimated using average intensity of the spots identified within the circular region. For each condition 1400-9000 cells were imaged in total in at least three independent experiments.

\section{Identification of functional clusters}

The STRING database was used to identify known and predicted protein-protein interactions between the hits $(\mathrm{v} 8.3)^{13}$. Only interactions with scores above 0.7 are reported here.

\section{Alexa $^{488}$-labelled transferrin uptake assay}

siRNA-transfected or BafA1-treated cells were incubated in serum-free DMEM for $45 \mathrm{~min}$ prior to a 7 min pulse with Alexa ${ }^{488}$-labelled transferrin at $37^{\circ} \mathrm{C}$. The cells were then washed and fixed ( $3.3 \%$ formaldehyde, $15 \mathrm{~min}$ ) at $4^{\circ} \mathrm{C}$, surface transferrin receptor was labelled using an Alexa ${ }^{647}$ secondary antibody, and the DNA was stained with Hoechst. Fluorescence data were collected using the plate reader as described above. The Alexa ${ }^{488}$ / Alexa $^{647}$ and Alexa ${ }^{647} /$ Hoechst ratios were used as a measure of endocytosis efficiency and transferrin receptor accumulation at the cell surface respectively. For the knockdown experiments, the ratios were normalised per experimental repeat by dividing by the mean value of the RISC-free control wells. For time course experiments, triplicates within an experiment were averaged (Alexa ${ }^{488} /$ Hoechst and Alexa ${ }^{647} /$ Hoechst ratios calculated for each well) and for each time point, the values from the BafA1-treated wells were normalised 
to the mean of the wells treated with DMSO for the same length of time. The Alexa ${ }^{488}$ / Alexa ${ }^{647}$ ratios were then calculated by dividing the normalised values.

\section{${ }^{125}$ I-labeled transferrin uptake assay}

The ${ }^{125}$ I-transferrin uptake assay has been previously described ${ }^{31}$. Briefly, control and treated cells were pre-incubated on ice with ${ }^{125} \mathrm{I}$-labeled transferrin in serum-free medium for $30 \mathrm{~min}$ at $4{ }^{\circ} \mathrm{C}$, followed by incubations at $37^{\circ} \mathrm{C}$ for various times. The medium was collected, surface-bound transferrin was stripped with a low $\mathrm{pH}$ wash, and the cells were extracted with $1 \mathrm{M} \mathrm{NaOH}$. Counts were quantified with a gamma counter. The graphs represent counts associated with intracellular transferrin as a fraction of total.

\section{Fluorescence microscopy}

For FRAP experiments, the cells were transfected with clathrin light chain A-GFP, BafA1treated $2 \mathrm{~h}$ post transfection, and imaged $24 \mathrm{~h}$ later in $\mathrm{CO}_{2}$-independent medium (Invitrogen) with a Zeiss LSM710 microscope equipped with a temperature controlled incubator $\left(37^{\circ} \mathrm{C}\right)$. Fluorescence intensity data were collected in the bleach region (ROI1), a reference region within the same cell (ROI2), and a background region (ROI3). The measurements were performed at $2 \mathrm{~s}$ intervals for $180 \mathrm{~s}$, with the beach following the $5^{\text {th }}$ readout. Bleaching resulted in average loss of $73 \%$ and $83 \%$ of fluorescence for control and BafA1-treated samples respectively. The data for ROI1 was corrected for the background and photobleaching $\left(\mathrm{F}_{\mathrm{ROI} 1}-\mathrm{F}_{\mathrm{ROI} 3}\right) /\left(\mathrm{F}_{\mathrm{RO} 2}-\mathrm{F}_{\mathrm{ROI} 3}\right)$, the fluorescence of the first post-bleach readout was subtracted from the measurements, and the results were divided by the average fluorescence of the first five time points. Three independent experiments were performed; in each from 9 to 18 cells per condition were analyzed.

For filipin staining, the cells were fixed for $30 \mathrm{~min}$ in $3.3 \%$ formaldehyde/PBS and incubated with filipin for $2 \mathrm{~h}$. Alexa ${ }^{488}$ transferrin uptake for microscopy was performed for $7 \mathrm{~min}$ at $37^{\circ} \mathrm{C}$ and the cells were fixed in $3.3 \%$ formaldehyde for $15 \mathrm{~min}$. Images were acquired using a Zeiss Axiovert 200M widefield fluorescence microscope equipped with a Roper Scientific CoolSNAP cf camera and a Plan-Apo 63x/1.4 oil-immersion lens using OpenLab software. The panels were assembled using Adobe Photoshop CS4.

\section{Resin embedding of whole cells}

For thin section electron microscopy, control, V-ATPase depleted cells (using ATP6V0C and ATP6V1B2 SMARTpools) and BafA1-treated (16 hr) cells were fixed in tissue culture dishes and prepared for EM as previously described ${ }^{30}$. Ultrathin sections were cut, collected onto formvar/carbon-coated EM grids, and stained with uranyl acetate and lead citrate. The sections were observed in a Philips CM 100 transmission electron microscope (Philips Electron Optics) at $80 \mathrm{kV}$.

For quantitative analysis of the size of CCSs, micrographs were taken of cells sectioned perpendicular to the PM at a magnification of $\times 34,000$ using a digital camera (Megaview III TEM; Soft Imaging System). For CCSs that were in continuity with the PM, the depth of the pit and the width of the neck were measured, whereas for cross-sectioned CCSs (within 250 $\mathrm{nm}$ of the PM), two axes were measured using iTEM software. For each condition, at least 93 CCSs were scored.

\section{PM replicas}

Control and BafA1-treated cells ( $24 \mathrm{~h}$ ), with or without $12.5 \mu \mathrm{g} / \mathrm{ml}$ cholesterol in the media, were grown on glass coverslips, washed in warm PBS, incubated for several seconds with polylysine in PBS and 'unroofed' using sonication as described ${ }^{67}$, except that sonication was performed in warm Hank's solution (HBSS). The exposed PMs were immediately fixed 
in $2 \%$ gluteraldehyde in $25 \mathrm{mM}$ HEPES, $125 \mathrm{mM} \mathrm{K}$-acetate, $50 \mathrm{mM}$-Mg-acetate, $\mathrm{pH} 7.3$ and further processed by critical point drying and rotary shadowing, as described ${ }^{33}$ and viewed in a Tecnai Spirit Biotwin $120 \mathrm{keV}$ TEM (FEI Company). The images were acquired with a Gatan Orius CCD camera.

\section{pH measurements}

For $\mathrm{pH}$ measurements, cells were transfected with $\mathrm{pH}$ constructs $24 \mathrm{~h}$ prior to being assayed. The cells were harvested in either $10 \%$ FCS/PBS or pH buffers $(15 \mathrm{mM} \mathrm{NaOH}, 10 \mathrm{mM}$ PIPES, $10 \mathrm{mM}$ HEPES, $5 \mathrm{mM}$ Glucose, $1 \mathrm{mM} \mathrm{NaH}_{2} \mathrm{PO}_{4}, 0.5 \mathrm{mM} \mathrm{MgSO}_{4}, 1 \mathrm{mM} \mathrm{CaCl}_{2}, 50$ $\mathrm{mM} \mathrm{KCl}$ and $90 \mathrm{mM}$ potassium L-glutamate brought to required $\mathrm{pH}$ using $\mathrm{KCl}$ or $\mathrm{NaOH}$ ) and analysed on the BD LSRFortessa (BD Biosciences) Flow Cytometer using a $488 \mathrm{~nm}$ laser with 430/30 filters to excite SEpHluorin and a $561 \mathrm{~nm}$ laser with 610/20 filters to excite mCherry.

Moderate expressors gated in the mCherry channel were selected for analysis. For quantification, mCherry and pHluorin were background-corrected, and the SEpHluorin/ mCherry ratio was calculated (using the 'derived parameter' function in FlowJo Version 7.6). Then the median values of the ratio from BafA1, DMSO or $\mathrm{pH}$ buffer-treated cells were normalised to untreated cells. Two-tailed, unpaired t-test was used to estimate statistical significance.

\section{Statistical methods}

Details of statistical analyses are provided in subsections referring to the individual assays.

\section{Data access}

The microarray data were deposited in GEO database (GSE39954). The MIARE-compatible screening data can be downloaded from: http://www.bioinformatics.cimr.cam.ac.uk/siRNA/ Robinson_Screen.

\section{Supplementary Material}

Refer to Web version on PubMed Central for supplementary material.

\section{Acknowledgments}

We would like to thank Michael Boutros (DKFZ) for providing the siRNA reannotation data, Sue Arden (CIMR) for mRNA isolation, Julien Bauer (Centre for Microarray Resources, Department of Pathology, Cambridge) for analysis of the microarray data, Sergio Grinstein (University of Toronto) for the $\mathrm{pH}$ sensor plasmids and helpful suggestions, Jeremy Skepper (Multi-Imaging Centre, Department of Anatomy, University of Cambridge) for help with the critical point drying, all members of the Robinson lab, in particular Georg Borner, for invaluable discussions, and Paul Luzio and John Kilmartin for reading the manuscript and helpful suggestions. This work was funded by the Wellcome Trust.

\section{REFERENCES}

1. Pelkmans L, et al. Genome-wide analysis of human kinases in clathrin- and caveolae/raft-mediated endocytosis. Nature. 2005; 436:78-86. [PubMed: 15889048]

2. Snijder B, et al. Population context determines cell-to-cell variability in endocytosis and virus infection. Nature. 2009; 461:520-523. [PubMed: 19710653]

3. Collinet C, et al. Systems survey of endocytosis by multiparametric image analysis. Nature. 2010; 464:243-249. [PubMed: 20190736]

4. Balklava Z, Pant S, Fares H, Grant BD. Genome-wide analysis identifies a general requirement for polarity proteins in endocytic traffic. Nat. Cell Biol. 2007; 9:1066-1073. [PubMed: 17704769] 
5. Kozik P, Francis RW, Seaman MN, Robinson MS. A screen for endocytic motifs. Traffic. 2010; 11:843-855. [PubMed: 20214754]

6. Donaldson JG, Williams DB. Intracellular assembly and trafficking of MHC class I molecules. Traffic. 2009; 10:1745-1752. [PubMed: 19761542]

7. Zhang XD. Novel analytic criteria and effective plate designs for quality control in genome-scale RNAi screens. J. Biomol. Screen. 2008; 13:363-377. [PubMed: 18567841]

8. Keyel PA, et al. A single common portal for clathrin-mediated endocytosis of distinct cargo governed by cargo-selective adaptors. Mol. Biol. Cell. 2006; 17:4300-4317. [PubMed: 16870701]

9. Jackson A, et al. Position-specific chemical modification of siRNAs reduces "off-target" transcript silencing. RNA. 2006; 12:1197-1205. [PubMed: 16682562]

10. Birmingham A, et al. $3^{\prime}$ UTR seed matches, but not overall identity, are associated with RNAi offtargets. Nat. Methods. 2006; 3:199-204. [PubMed: 16489337]

11. Tebar F, Bohlander SK, Sorkin A. Clathrin assembly lymphoid myeloid leukemia (CALM) protein: localization in endocytic-coated pits, interactions with clathrin, and the impact of overexpression on clathrin-mediated traffic. Mol. Biol. Cell. 1999; 10:2687-2702. [PubMed: 10436022]

12. Hinrichsen L, Harborth J, Andrees L, Weber K, Ungewickell EJ. Effect of clathrin heavy chainand alpha-adaptin-specific small inhibitory RNAs on endocytic accessory proteins and receptor trafficking in HeLa cells. J. Biol. Chem. 2003; 278:45160-45170. [PubMed: 12960147]

13. von Mering C, et al. STRING: known and predicted protein-protein associations, integrated and transferred across organisms. Nucleic Acids Res. 2005; 33:D433-437. [PubMed: 15608232]

14. Johnson LS, Dunn KW, Pytowski B, McGraw TE. Endosome acidification and receptor trafficking: bafilomycin A1 slows receptor externalization by a mechanism involving the receptor's internalization motif. Mol. Biol. Cell. 1993; 4:1251-1266. [PubMed: 8167408]

15. Brown D, Paunescu TG, Breton S, Marshansky V. Regulation of the V-ATPase in kidney epithelial cells: dual role in acid-base homeostasis and vesicle trafficking. J. Exp. Biol. 2009; 212:17621772. [PubMed: 19448085]

16. Heuser J. Effects of cytoplasmic acidification on clathrin lattice morphology. J. Cell. Biol. 1989; 108:401-411. [PubMed: 2563729]

17. Heuser JE, Anderson RG. Hypertonic media inhibit receptor-mediated endocytosis by blocking clathrin-coated pit formation. J. Cell. Biol. 1989; 108:389-400. [PubMed: 2563728]

18. Wu X, et al. Clathrin exchange during clathrin-mediated endocytosis. J. Cell. Biol. 2001; 155:291300. [PubMed: 11604424]

19. Koivusalo M, et al. Amiloride inhibits macropinocytosis by lowering submembranous $\mathrm{pH}$ and preventing Rac1 and Cdc42 signaling. J. Cell. Biol. 2010; 188:547-563. [PubMed: 20156964]

20. Cosson P, de Curtis I, Pouysségur J, Griffiths G, Davoust J. Low cytoplasmic pH inhibits endocytosis and transport from the trans-Golgi network to the cell surface. J. Cell. Biol. 1989; 108:377-387. [PubMed: 2918022]

21. Mellman I, Fuchs R, Helenius A. Acidification of the endocytic and exocytic pathways. Annu. Rev. Biochem. 1986; 55:663-700. [PubMed: 2874766]

22. Chapman RE, Munro S. Retrieval of TGN proteins from the cell surface requires endosomal acidification. EMBO J. 1994; 13:2305-2312. [PubMed: 8194522]

23. Subtil A, et al. Acute cholesterol depletion inhibits clathrin-coated pit budding. Proc. Natl. Acad. Sci. USA. 1999; 96:6775-6780. [PubMed: 10359788]

24. Rodal SK, et al. Extraction of cholesterol with methyl-beta-cyclodextrin perturbs formation of clathrin-coated endocytic vesicles. Mol. Biol. Cell. 1999; 10:961-974. [PubMed: 10198050]

25. Hölttä-Vuori M, Tanhuanpää K, Möbius W, Somerharju P, Ikonen E. Modulation of cellular cholesterol transport and homeostasis by Rab11. Mol. Biol. Cell. 2002; 13:3107-3122. [PubMed: 12221119]

26. Furuchi T, Aikawa K, Arai H, Inoue K. Bafilomycin A1, a specific inhibitor of vacuolar-type $\mathrm{H}(+)$-ATPase, blocks lysosomal cholesterol trafficking in macrophages. J. Biol. Chem. 1993; 268:27345-27348. [PubMed: 8262974] 
27. Lange Y, Ye J, Steck TL. Circulation of cholesterol between lysosomes and the plasma membrane. J. Biol. Chem. 1998; 273:18915-18922. [PubMed: 9668068]

28. Taylor M, Perrais D, Merrifield CJ. A high precision survey of the molecular dynamics of mammalian clathrin-mediated endocytosis. PLoS Biol. 2011; 9:e1000604. [PubMed: 21445324]

29. Saffarian S, Cocucci E, Kirchhausen T. Distinct dynamics of endocytic clathrin-coated pits and coated plaques. PLoS Biol. 2009; 7:e1000191. [PubMed: 19809571]

30. Hirst J, et al. Auxilin depletion causes self-assembly of clathrin into membraneless cages in vivo. Traffic. 2008; 9:1354-1371. [PubMed: 18489706]

31. Motley A, Bright NA, Seaman MN, Robinson MS. Clathrin-mediated endocytosis in AP-2depleted cells. J. Cell. Biol. 2003; 162:909-918. [PubMed: 12952941]

32. Borner GH, et al. Multivariate proteomic profiling identifies novel accessory proteins of coated vesicles. J. Cell. Biol. 2012; 197:141-160. [PubMed: 22472443]

33. Meyerholz A, et al. Effect of clathrin assembly lymphoid myeloid leukemia protein depletion on clathrin coat formation. Traffic. 2005; 6:1225-1234. [PubMed: 16262731]

34. Huang F, Khvorova A, Marshall W, Sorkin A. Analysis of clathrin-mediated endocytosis of epidermal growth factor receptor by RNA interference. J. Biol. Chem. 2004; 279:16657-16661. [PubMed: 14985334]

35. Ball CL, Hunt SP, Robinson MS. Expression and localization of alpha-adaptin isoforms. J. Cell Sci. 1995; 108(Pt 8):2865-2875. [PubMed: 7593326]

36. Jackson AP, Seow HF, Holmes N, Drickamer K, Parham P. Clathrin light chains contain brainspecific insertion sequences and a region of homology with intermediate filaments. Nature. 1987; 326:154-159. [PubMed: 3821891]

37. Cao H, Garcia F, McNiven MA. Differential distribution of dynamin isoforms in mammalian cells. Mol. Biol. Cell. 1998; 9:2595-2609. [PubMed: 9725914]

38. McNiven MA, Cao H, Pitts KR, Yoon Y. The dynamin family of mechanoenzymes: pinching in new places. Trends Biochem. Sci. 2000; 25:115-120. [PubMed: 10694881]

39. Hofmann JC, Husedzinovic A, Gruss OJ. The function of spliceosome components in open mitosis. Nucleus. 2010; 1:447-459. [PubMed: 21327086]

40. Fielding AB, Willox AK, Okeke E, Royle SJ. Clathrin-mediated endocytosis is inhibited during mitosis. Proc. Natl. Acad. Sci. USA. 2012

41. Choudhary $\mathrm{C}$, et al. Lysine acetylation targets protein complexes and co-regulates major cellular functions. Science. 2009; 325:834-840. [PubMed: 19608861]

42. Gallant P. Control of transcription by Pontin and Reptin. Trends Cell Biol. 2007; 17:187-192. [PubMed: 17320397]

43. Sigala B, Edwards M, Puri T, Tsaneva IR. Relocalization of human chromatin remodeling cofactor TIP48 in mitosis. Exp. Cell Res. 2005; 310:357-369. [PubMed: 16157330]

44. Lee HJ, Chun M, Kandror KV. Tip60 and HDAC7 interact with the endothelin receptor a and may be involved in downstream signaling. J. Biol. Chem. 2001; 276:16597-16600. [PubMed: 11262386]

45. Shepard BD, Tuma DJ, Tuma PL. Lysine acetylation induced by chronic ethanol consumption impairs dynamin-mediated clathrin-coated vesicle release. Hepatology. 2011

46. Infante RE, et al. NPC2 facilitates bidirectional transfer of cholesterol between NPC1 and lipid bilayers, a step in cholesterol egress from lysosomes. Proc. Natl. Acad. Sci. USA. 2008; 105:15287-15292. [PubMed: 18772377]

47. Deffieu MS, Pfeffer SR. Niemann-Pick type C 1 function requires lumenal domain residues that mediate cholesterol-dependent NPC2 binding. Proc. Natl. Acad. Sci. USA. 2011; 108:1893218936. [PubMed: 22065762]

48. Lange Y, Ye J, Rigney M, Steck T. Cholesterol movement in Niemann-Pick type C cells and in cells treated with amphiphiles. J. Biol. Chem. 2000; 275:17468-17475. [PubMed: 10751394]

49. Lange Y, Ye J, Rigney M, Steck TL. Dynamics of lysosomal cholesterol in Niemann-Pick type C and normal human fibroblasts. J. Lipid Res. 2002; 43:198-204. [PubMed: 11861661]

50. Liscum L. Niemann-Pick type C mutations cause lipid traffic jam. Traffic. 2000; 1:218-225. [PubMed: 11208105] 
51. Steck TL, Ye J, Lange Y. Probing red cell membrane cholesterol movement with cyclodextrin. Biophys. J. 2002; 83:2118-2125. [PubMed: 12324429]

52. Bruckner R, Mansy S, Ricardo A, Mahadevan L, Szostak J. Flip-flop-induced relaxation of bending energy: implications for membrane remodeling. Biophys. J. 2009; 97:3113-3122. [PubMed: 20006948]

53. Liu K, Surendhran K, Nothwehr SF, Graham TR. P4-ATPase requirement for AP-1/clathrin function in protein transport from the trans-Golgi network and early endosomes. Mol. Biol. Cell. 2008; 19:3526-3535. [PubMed: 18508916]

54. Pérez-Sayáns M, Somoza-Martín JM, Barros-Angueira F, Rey JM, García-García A. V-ATPase inhibitors and implication in cancer treatment. Cancer treatment reviews. 2009; 35:707-713. [PubMed: 19758758]

55. Straud S, Zubovych I, De Brabander J, Roth M. Inhibition of iron uptake is responsible for differential sensitivity to V-ATPase inhibitors in several cancer cell lines. PLoS ONE. 2010; 5:e11629. [PubMed: 20661293]

56. Yan Y, Denef N, Schüpbach T. The vacuolar proton pump, V-ATPase, is required for notch signaling and endosomal trafficking in Drosophila. Dev. Cell. 2009; 17:387-402. [PubMed: 19758563]

57. Vaccari T, Duchi S, Cortese K, Tacchetti C, Bilder D. The vacuolar ATPase is required for physiological as well as pathological activation of the Notch receptor. Development. 2010; 137:1825-1832. [PubMed: 20460366]

58. Hermle T, Saltukoglu D, Gr ${ }^{\natural}$ newald J, Walz G, Simons M. Regulation of Frizzled-dependent planar polarity signaling by a V-ATPase subunit. Curr. Biol. 2010; 20:1269-1276. [PubMed: 20579879]

59. Cruciat CM, et al. Requirement of prorenin receptor and vacuolar H+-ATPase-mediated acidification for Wnt signaling. Science. 2010; 327:459-463. [PubMed: 20093472]

60. Windler SL, Bilder D. Endocytic internalization routes required for delta/notch signaling. Curr. Biol. 2010; 20:538-543. [PubMed: 20226669]

61. Seugnet L, Simpson P, Haenlin M. Requirement for dynamin during Notch signaling in Drosophila neurogenesis. Dev. Biol. 1997; 192:585-598. [PubMed: 9441691]

62. Gagliardi M, Piddini E, Vincent JP. Endocytosis: a positive or a negative influence on Wnt signalling? Traffic. 2008; 9:1-9. [PubMed: 17908284]

63. Yu A, et al. Association of Dishevelled with the clathrin AP-2 adaptor is required for Frizzled endocytosis and planar cell polarity signaling. Dev. Cell. 2007; 12:129-141. [PubMed: 17199046]

64. Tiwari RK, Kusari J, Sen GC. Functional equivalents of interferon-mediated signals needed for induction of an mRNA can be generated by double-stranded RNA and growth factors. EMBO J. 1987; 6:3373-3378. [PubMed: 2828026]

65. Lin SM, Du P, Huber W, Kibbe WA. Model-based variance-stabilizing transformation for Illumina microarray data. Nucleic Acids Res. 2008; 36:e11. [PubMed: 18178591]

66. Du P, Kibbe WA, Lin SM. lumi: a pipeline for processing Illumina microarray. Bioinformatics. 2008; 24:1547-1548. [PubMed: 18467348]

67. Heuser J. The production of 'cell cortices' for light and electron microscopy. Traffic. 2000; 1:545552. [PubMed: 11208142]

68. Zhang XD, et al. The use of strictly standardized mean difference for hit selection in primary RNA interference high-throughput screening experiments. J. Biomol. Screen. 2007; 12:497-509.

[PubMed: 17435171] 


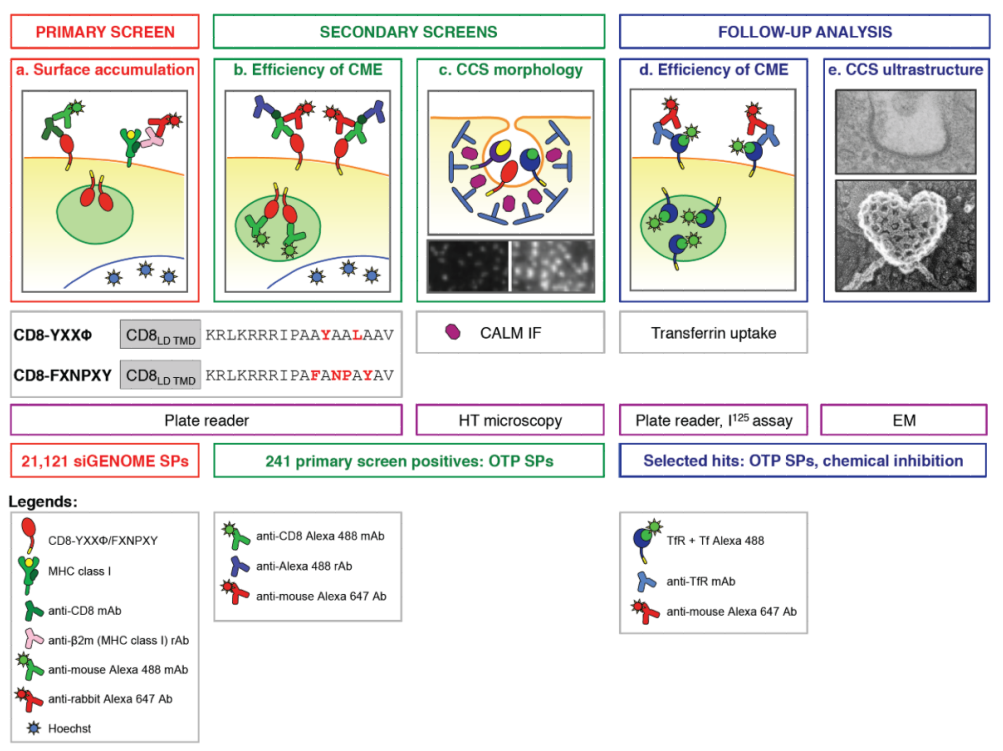

Figure 1. Summary of the multi-step screening strategy

In the primary genome-wide screen (a), 21,121 siGENOME SMARTpools (four siRNAs per gene) were screened using a plate reader-based assay for hits that lead to accumulation of clathrin-dependent cargo proteins on the cell surface, without increasing the levels of surface MHC class I (see also Figure 2). CD8 chimeras with YXXФ or FXNPXY motifs inserted into their cytoplasmic tails were used as model CCV cargo. 241 primary screen positives were selected for the secondary screens $(b, c)$. The efficiency of endocytosis was measured using the CD8 chimera-expressing cells (b, see also Figure 3) and the morphology of CCSs at the plasma membrane was assessed using a CALM immunolabelling and automated microscopy-based assay (c, see also Figure 4). For selected genes, knockdown cells were tested for the ability to internalize transferrin (d), and CCSs at the PM were characterized using electron microscopy (e). 
a

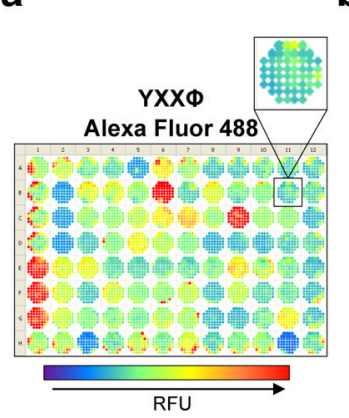

b

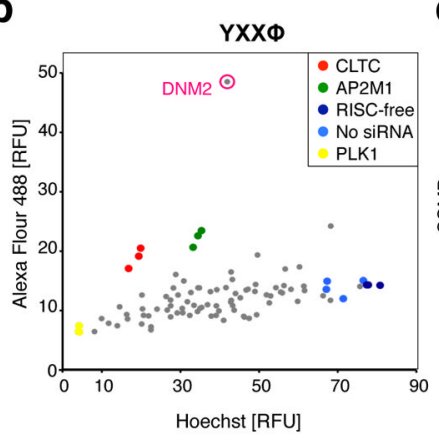

C C $\quad$ YXX $\Phi$

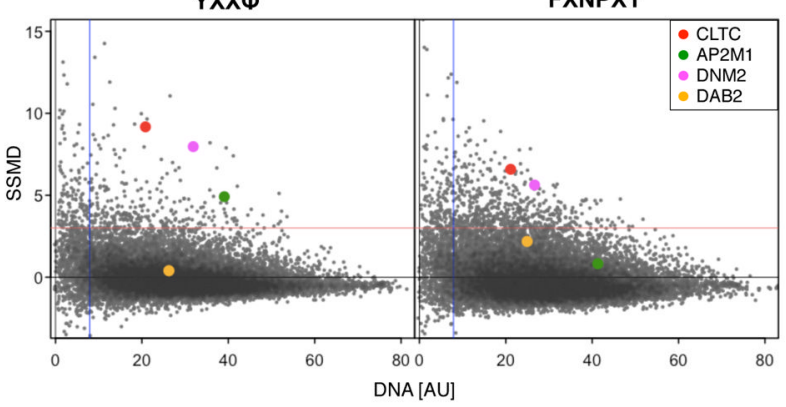

d
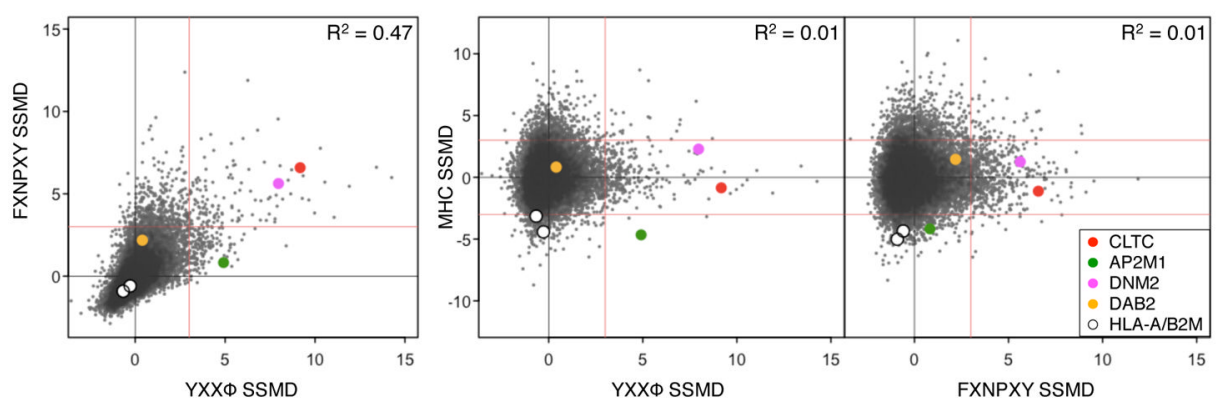

e

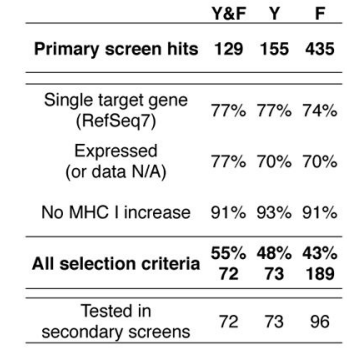

Figure 2. Genome-wide primary screen for surface accumulation of $\mathrm{CCV}$ cargo

(a) Cells transfected with the siRNAs were fixed after $72 \mathrm{~h}$ and stained for surface CD8 (Alexa ${ }^{488}$ ), surface MHC class I (Alexa ${ }^{647}$ ), and DNA (Hoechst). Using a plate reader, 52 points were scanned in each of the three fluorescence channels, here shown on a sample plate for Alexa ${ }^{488}$. The fluorescence intensity of each point is colour-coded according to the scale presented, and the corresponding plate map is presented in Supplementary Figure S1. (b) Alexa ${ }^{488}$ (surface CD8-YXXФ) and Hoechst readouts from one of the screening plates. Each point represents one well/SMARTpool. Control wells are colour-coded according to the legend and grey points represent the library wells. Similar plots for each tested SMARTpool can be found at http://www.bioinformatics.cimr.cam.ac.uk/siRNA/ Robinson_Screen. To analyse the data further, we calculated the ratio of Alexa ${ }^{488} /$ Hoechst fluorescence for each point and then normalized the ratios to the positive (CLTC) and negative (RISC-free, mock) controls (see Supplementary Figures S2,S3). (c) Screening results for the 21,121 SMARTpools. For each pool, the strength (normalized Alexa ${ }^{488}$ / Hoechst ratio of the duplicates) and reproducibility were scored using SSMD (see Supplementary Figure S4). The red line indicates the threshold for hit selection (SSMD > 3) and the blue line the threshold for cell viability. SMARTpools against CLTC, AP2M1, DNM2, and DAB2 present in the library are colour-coded. (d) Correlation between the phenotypes for the different types of membrane proteins: CD8-YXX $\Phi$, CD8-FXNPXY, and endogenous MHC-I. (e) Number and/or percentage of SMARTpools that fulfilled the different hit selection criteria for both YXXФ- and FXNPXY-cargo (Y\&F), or for one of the cargos only (Y: YXXФ, F: FXNPXY). SMARTpools with SSMD > 3 were selected as primary screen positives and analyzed for sequence specificity according to RefSeq 25 , mRNA expression (Supplementary Table S3), and phenotype specificity (increase on the cell surface specific for CCV-cargo only, not accompanied by an increase of MHC-I). 
a

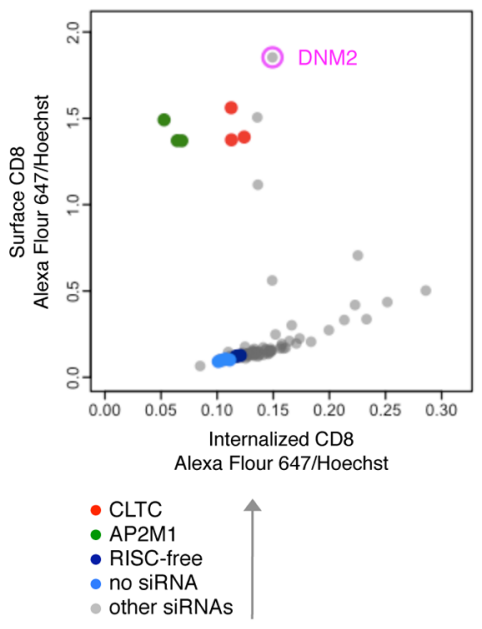

b

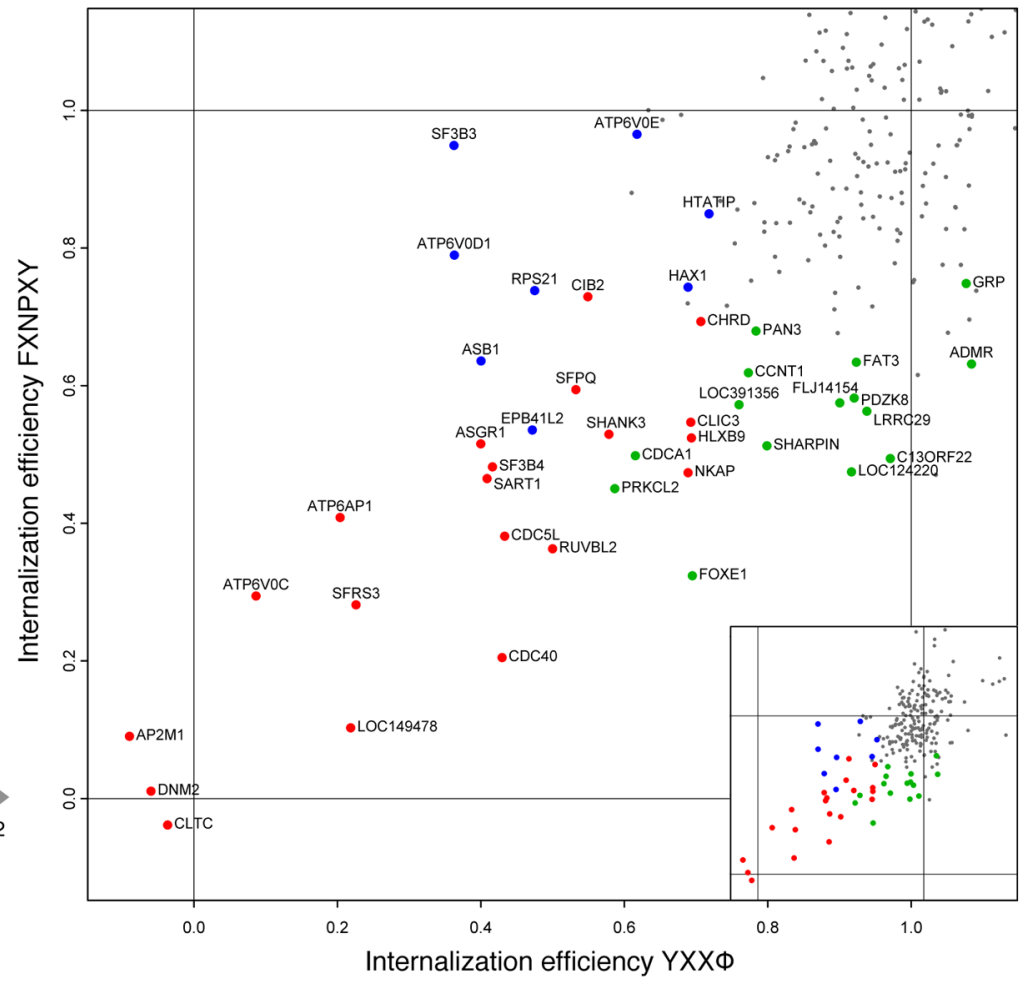

Figure 3. Secondary screen for efficiency of CME

The siRNA-transfected cells were incubated for $40 \mathrm{~min}$ in the continuous presence of Alexa ${ }^{488}$-labelled anti-CD8 and fixed. Surface CD8 was relabelled with Alexa ${ }^{647}$ secondary antibody and surface CD8-associated Alexa ${ }^{488}$ was quenched with an anti-Alexa ${ }^{488}$ antibody (see Figure 1b). (a) Data from a sample plate: surface (Alexa ${ }^{647} /$ Hoechst) and internal (Alexa ${ }^{488} /$ Hoechst) CD8-YXXФ. Control wells are colour-coded according to the legend, and DNM2 is highlighted as an example of a hit. Similar plots for each tested SMARTpool can be found online (see legend to Figure 2b). (b) Normalized endocytosis efficiency of CD8-YXXФ and CD8-FXNPXY in knockdown cells. Values close to one correspond to the uptake efficiency in the "no knockdown" control cells, values around zero correspond to uptake efficiency in CLTC knockdown control wells. Hits that decrease efficiency of endocytosis (SSMD 22) of CD8-YXXФ and CD8-FXNPXY are highlighted in red, and cargo-specific hits in blue (YXXФ) and green (FXNPXY). 
a

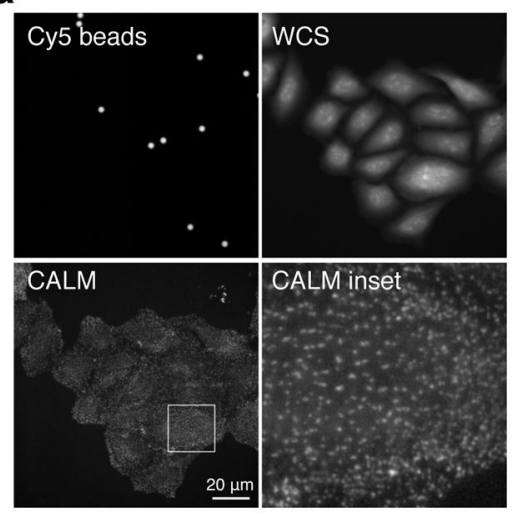

d
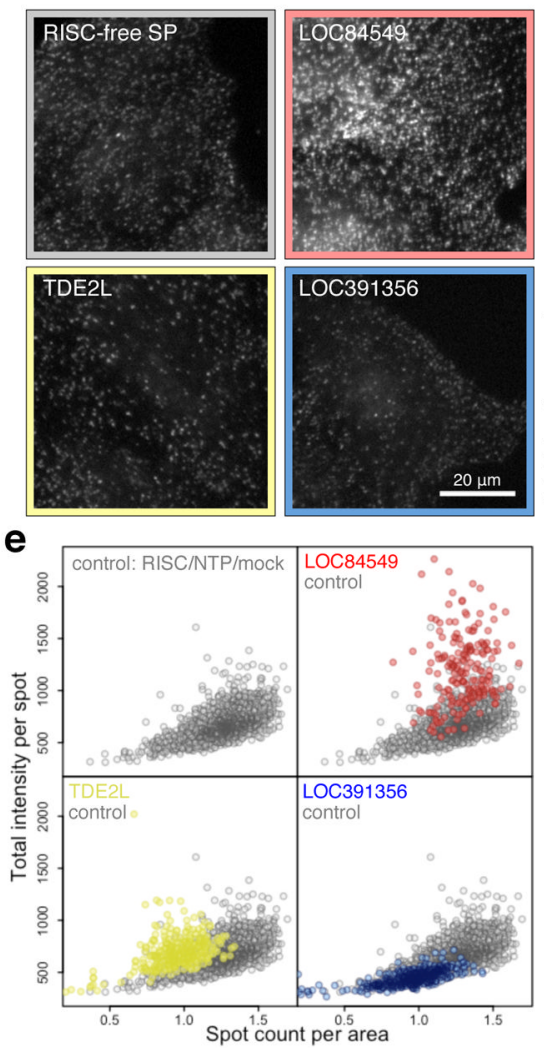

LOC391356 b
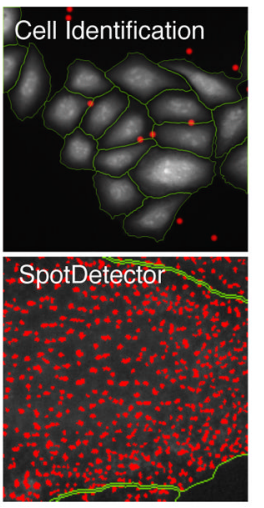

f

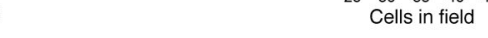

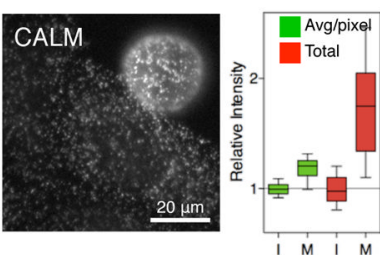
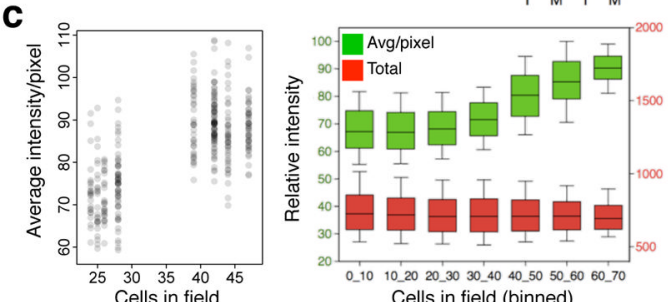
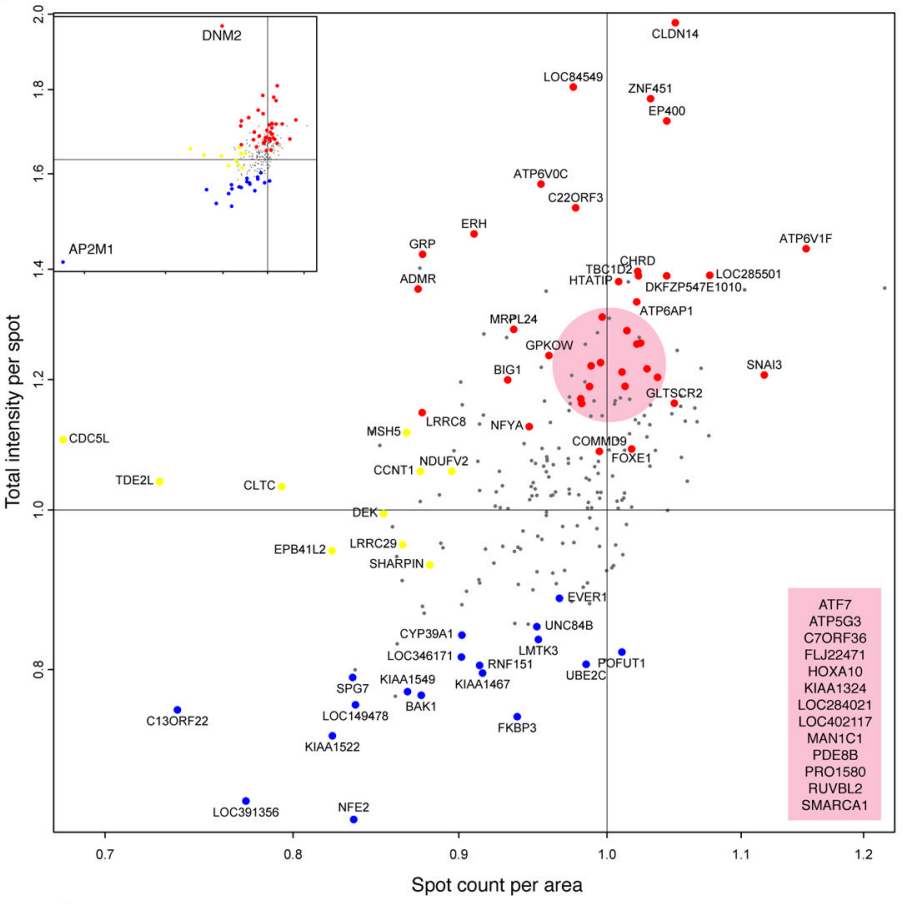

- SSMD (Total intensity per spot) $\geq 3$
- SSMD (Total intensity per spot) $\leq-3$
SSMD (Spot count per area) $\leq-3$

Figure 4. Secondary screen to analyze morphology of CCSs at the PM

(a) CALM immunolabelling assay. Cy5 beads were used as a focussing reference. Green lines indicate cell boundaries and red spots indicate CALM-labelled CCSs. (b) CALM labelling in interphase and mitotic cells. Normalized (per field) average per pixel and total fluorescence intensity of CALM-labelled spots in interphase $(\mathrm{I}, \mathrm{n}=286)$ and mitotic $(\mathrm{M}, \mathrm{n}=$ 26 ) cells. Box: median, the $25^{\text {th }}$ and $75^{\text {th }}$ percentiles, whiskers: $10^{\text {th }}-90^{\text {th }}$ percentiles. Mitotic cells were excluded from the final analysis because of effects on spot fluorescence intensity. (c) CALM labelling in fields with low and high cell density. Scatter plot represents average intensity per pixel of CCSs from fields (within a single well) with lowest and highest density (one dot per cell). Bar chart shows average intensity per pixel and total intensity per spot from RISC-free wells, data from 45 wells, 15 plates over two independent 
experiments. Cells $(\mathrm{n}=16652)$ are grouped according to cell number per field. Box: median, the $25^{\text {th }}$ and $75^{\text {th }}$ percentiles, whiskers: $10^{\text {th }}-90^{\text {th }}$ percentiles. Average intensity is higher in high density fields, which may correlate with the observation that CME is more active in such regions ${ }^{2}$. To ensure phenotype differences were not due to cell density, fields containing > 30 cells were not analyzed. (d) CALM images from the three phenotypic groups defined: increased total spot brightness (red), decreased total spot brightness (blue), and decreased spot count, no change in brightness (yellow). (e) Representative data for each knockdown in (d). Each point represents a cell for which average total intensity per CALM spot and spot count per area were calculated: Coloured points: cells from a well with the indicated SMARTpool; grey points: cells from control wells on the same plate. Similar plots for each knockdown can be found online. (f) Normalized total fluorescence per CALMlabelled spot and spot count per area for genes tested in the secondary screens. Colour-coded points represent genes with SSMDs $>3$ for spot brightness (red), and SSMD $<-3$ for spot count (yellow) or brightness (blue). 


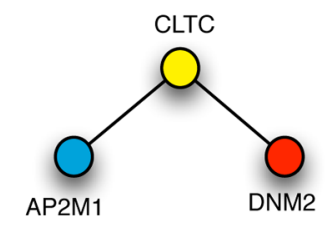

CCV coat components

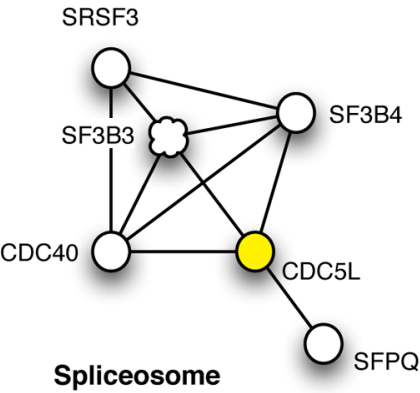

COLOUR

CALM labeled CCSs

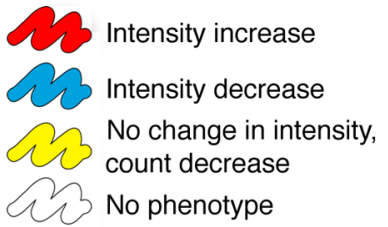

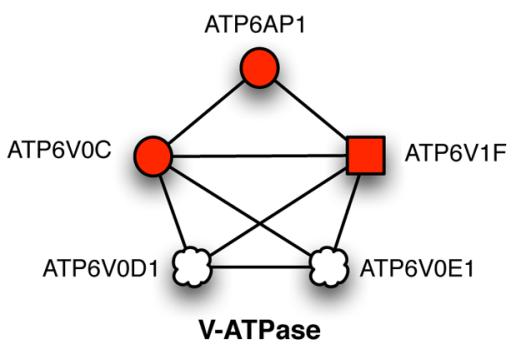

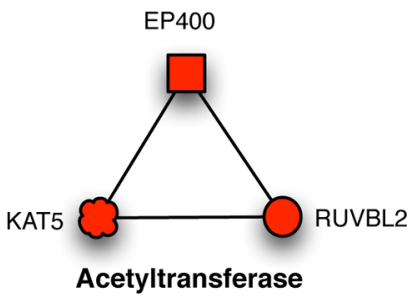

SHAPE

Endocytosis efficiency

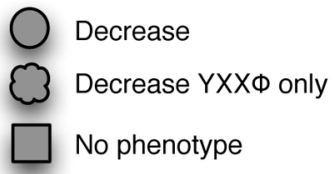

Figure 5. Functional clusters within the hits

Hits with a phenotype in either the endocytosis or the CCS morphology assay were pooled and analyzed using STRING. Four functional clusters were identified. Within each network, the colour of the symbol indicates the phenotype in the CCS morphology screen, and the shape of the symbol indicates the phenotype in the endocytosis efficiency screen. KAT5 is the official gene symbol for the gene annotated in the library as HTATIP. Although theoretically one would expect all of the genes encoding subunits of the same complex to have the same knockdown phenotype, in practice there are differences due to factors such as knockdown efficiency and threshold for hit selection. In addition, genes from the same functional cluster might have different regulatory effects on CCVs components, and therefore different effects on CCS intensity. 


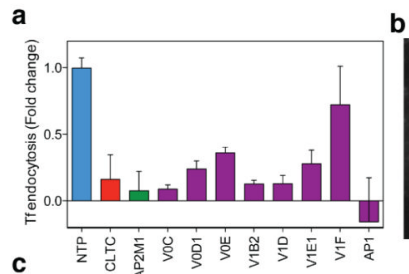

b

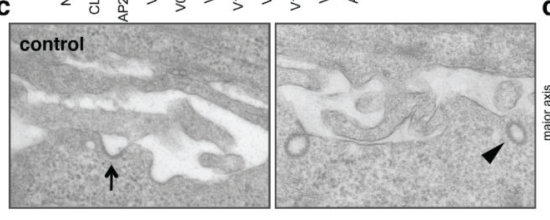

d
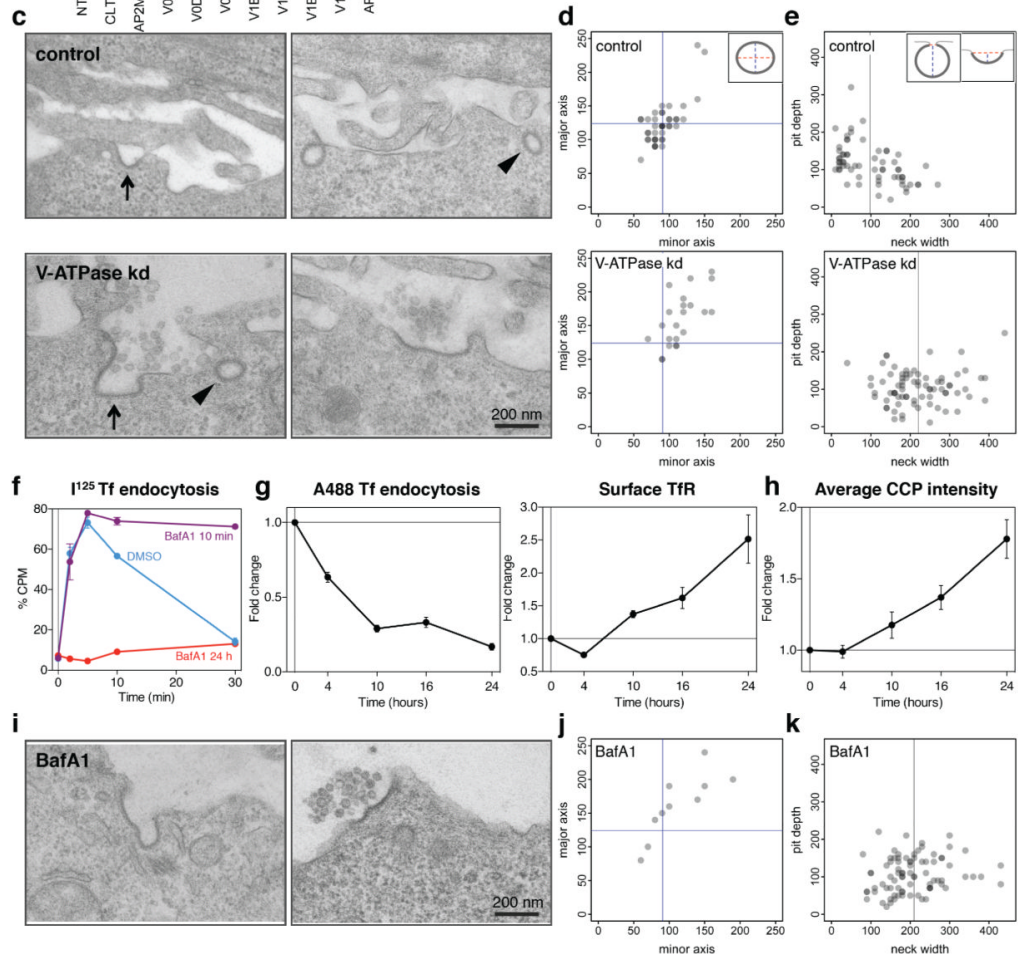

$\mathbf{k}$
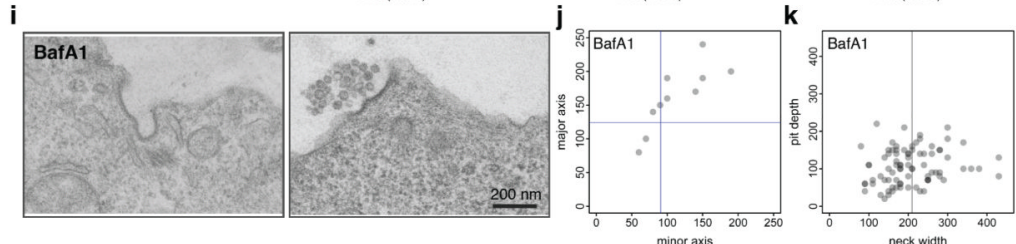

Figure 6. siRNA-mediated depletion of V-ATPase or prolonged inhibition with Bafilomycin A1 leads to accumulation of large, non-constricted CCSs at the PM

(a) Efficiency of transferrin internalization after siRNA-mediated depletion of V-ATPase subunits. The cells were incubated with Alexa ${ }^{488}$-transferrin for $7 \mathrm{~min}$, fixed, and surface transferrin receptor was stained using a secondary Alexa ${ }^{647}$ antibody. Alexa ${ }^{488} /$ Alexa $^{647}$ ratios from three independent experiments normalised to the NTP control are plotted. (b) CALM immunofluorescence in ATP6V0C or ATP6V1B2 knockdown cells. (c, d) EM analysis of control and V-ATPase knockdown cells (combined ATP6V0C and ATP6V1B2 SMARTpools). (c) Representative micrographs of CCSs at the PM. Arrows indicate examples of CCPs connected to the PM within the section, wedges indicate structures within $250 \mathrm{~nm}$ of the PM, likely to be PM-connected in a different $\operatorname{section}^{30}$ (d) Size distribution of sectioned CCSs within $250 \mathrm{~nm}$ from the PM (marked with wedges in (c)). The lengths of the major and minor axis were measured. Blue lines indicate means of CCSs in control cells. (e) Size distribution of CCPs connected to the PM within the section (marked with arrows in (c)). The pit depth and neck width were measured. Grey lines indicate mean neck width.(f) Kinetics of transferrin internalization in $24 \mathrm{~h}$ or 10 min BafA1-treated cells. Fraction of internalized ${ }^{125}$ I-transferrin is plotted for each time point. $\mathrm{n}=3$ independent experiments. (g) Efficiency of transferrin internalization and cell surface accumulation of transferrin receptor after pre-treatment with BafA1 for 4, 10, 16, and $24 \mathrm{~h}$. The assay was performed as described in (a). $n=3$ independent experiments. (h) Fold changes in the total spot intensity of CALM-labelled CCPs in a time course experiment with BafA1. ). $\mathrm{n}=3$ independent experiments. (i) EM analysis of the CCSs in cells treated with BafA1 for 24 h. (j) Size 
distribution of sectioned CCSs within $250 \mathrm{~nm}$ from the PM in BafA1-treated cells, analyzed as described for (d). (k) Size distribution of PM-connected CCPs in BafA1-treated cells, analyzed as described for (e). All error bars: \pm SEM. 

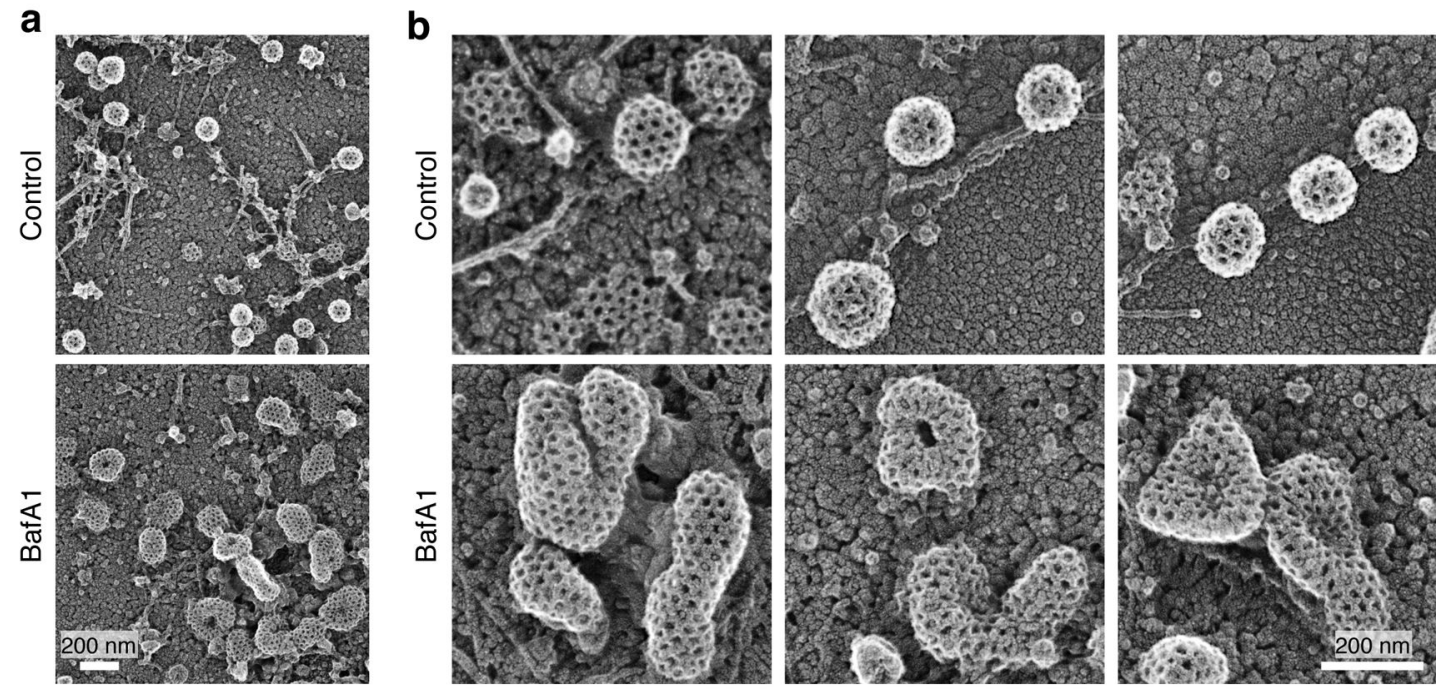

C

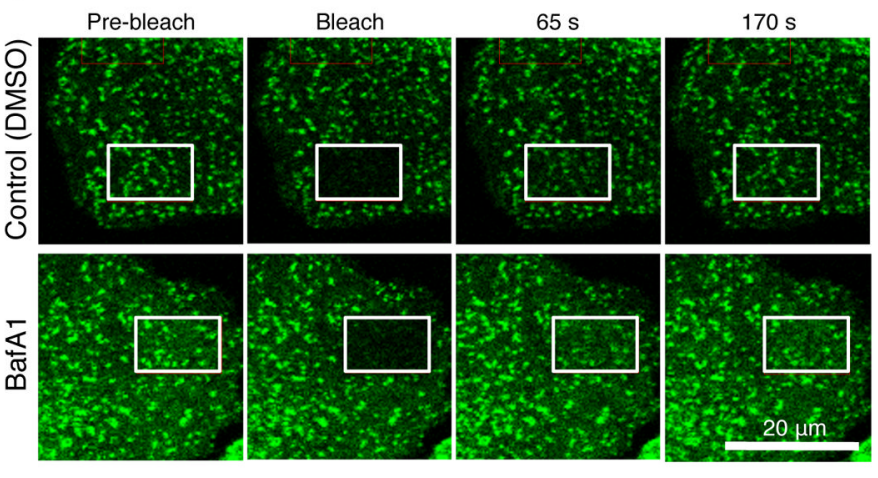

d

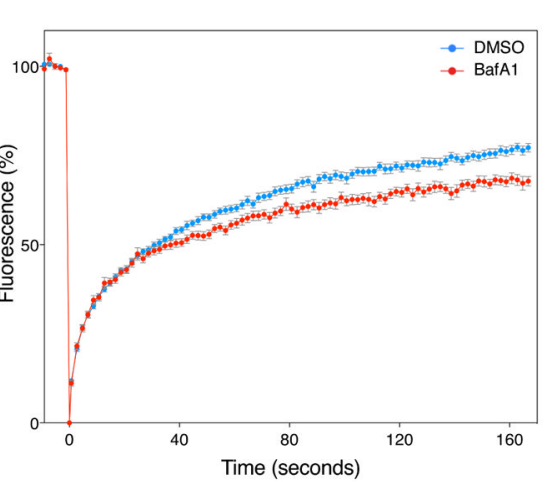

e

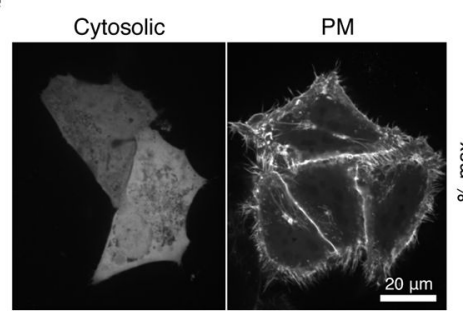

f
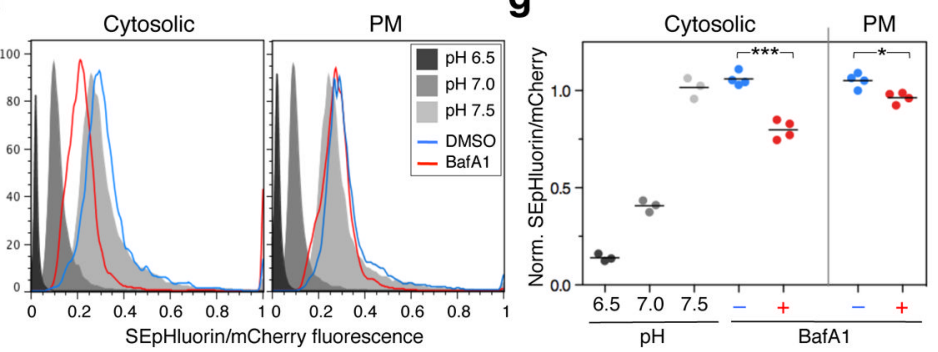

Figure 7. Inhibition of V-ATPase does not "lock" clathrin in microcages

$(\mathrm{a}, \mathrm{b})$ Plasma membrane replicas from control and BafA1 treated cells. The cells were "unroofed" by sonication, fixed, critical point dried, and rotary shadowed. Scale bars: 200 nm. (c, d) Clathrin dynamics at the PM were monitored in cells treated with BafA1 or DMSO by FRAP analysis of clathrin light chain-GFP. (c) Images of the FRAP region (white box) before and after photobleaching. (d) Corrected fluorescence recovery of the bleached region over time. Data from $n=3$ independent experiments, with 45 and 34 cells analyzed for DMSO and BafA1 respectively. Error bars: \pm SEM, $(e, f, g) ~ p H$ measurements in intact cells. (e) Localisation of the soluble and membrane-bound SEpHluorin/mCherry $\mathrm{pH}$ constructs (mCherry channel). (f) Cells transfected with the $\mathrm{pH}$ constructs were treated with BafA1 or DMSO for $24 \mathrm{~h}$, harvested in $20 \%$ FCS/PBS and analyzed by flow cytometry. For 
reference, untreated cells were harvested in $\mathrm{K}^{+} /$nigericin buffers of predetermined $\mathrm{pH}$. The histograms represent the pHluorin/mCherry fluorescence ratios. (g) Medians of SEpHluorin/ mCherry ratios normalised to the median of untreated cells in each experiment. *** $\mathrm{p} \leq$ $0.001, * \mathrm{p} \leq 0.05$. 

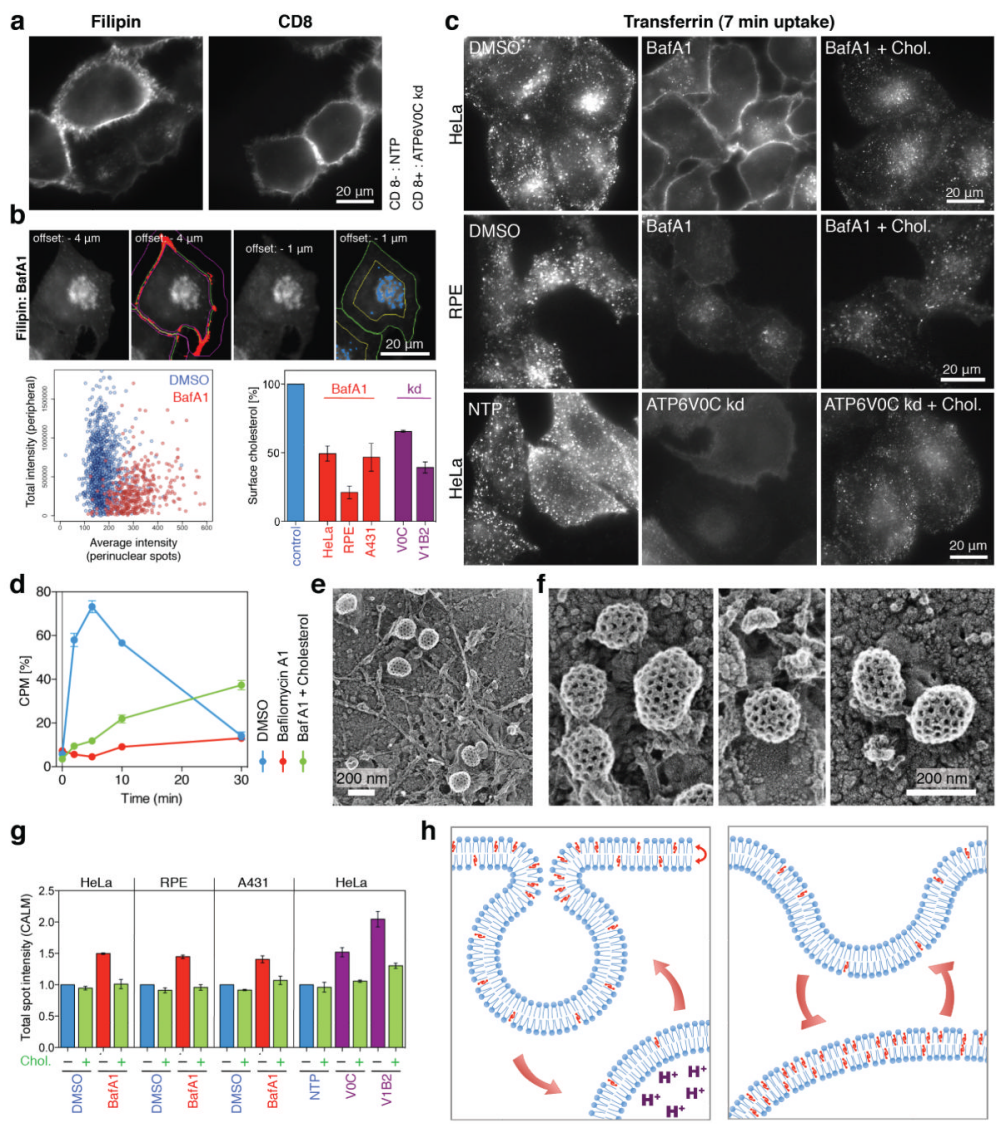

Figure 8. Soluble cholesterol rescues function and morphology of the PM CCPs in the VATPase-depleted cells

(a) Filipin staining in ATP6V0C knockdown $\left(\mathrm{CD}^{+}\right)$and NTP control (CD8 $\left.{ }^{-}\right)$cells. (b) Quantification of cholesterol depletion. Cholesterol was stained after a 24 h BafA1treatment or $72 \mathrm{~h}$ after V-ATPase depletion. WCS was used to identify cell boundaries (green lines). Images are at two focal planes: at the cell base (offset $-4 \mu \mathrm{m}$ ) to quantify fluorescence at the periphery (PM pool, red mask within the region enclosed the by purple lines) and in the middle (offset $-1 \mu \mathrm{m}$ ) to quantify intracellular fluorescence (blue spots within the perinuclear region, yellow line). (See Supplementary Figure S5). The scatter plot compares intracellular and PM filipin staining intensity in control (blue) and BafA1-treated (red) wells (each dot represents one cell). The bar graph shows levels of PM cholesterol for $\mathrm{n}=3$ independent experiments on BafA1-treated HeLa, RPE, and A431 cells, and after VATPase depletion (HeLa cells). BafA1 samples are normalized to DMSO, V-ATPase knockdowns to NTP controls. (c) Distribution of Alexa ${ }^{488}$-labelled transferrin after a 7 min incubation at $37^{\circ} \mathrm{C}$ in control, BafA1-treated, and BafA1-treated in the presence of $20 \mu \mathrm{g} / \mathrm{ml}$ soluble cholesterol cells. (d) Kinetics of ${ }^{125} \mathrm{I}$-transferrin uptake in control, BafA1-treated, and BafA1-treated in the presence of $20 \mu \mathrm{g} / \mathrm{ml}$ soluble cholesterol cells (see Figure 6e). The graph represents the fraction of internalized transferrin at different times. $n=3$ independent experiments. (e, f) PM replicas of BafA1-treated cells, grown in the presence of $12.5 \mu \mathrm{g} / \mathrm{ml}$ soluble cholesterol for $24 \mathrm{~h}$ (see Figure 7a, Supplementary Figure S8). (g) Total intensity of CALM-labelled spots (assay described in Figure 4). BafA1-treated samples were normalized to DMSO, and V-ATPase knockdowns to NTP controls. Where indicated, the growth medium contained $20 \mu \mathrm{g} / \mathrm{ml}$ cholesterol. $\mathrm{n}=3$ independent experiments. $* * \mathrm{p} \leq 0.01(\mathrm{~h})$ Model of cholesterol distribution and function in the presence/absence of a functional V- 
ATPase. Cholesterol flip-flop between leaflets of the bilayer reduces membrane tension generated in high curvature regions, such as CCP necks. Cholesterol is then recycled in vesicles from acidified endosomes back to the PM. Upon V-ATPase depletion or inhibition, endosomal $\mathrm{pH}$ increases, and cholesterol is retained. This causes PM cholesterol depletion and increases the energy needed to constrict CCP necks. All error bars: \pm SEM. 